\title{
Human DPP III - Keap1 Interactions: a Combined Experimental and Computational Study
}

\author{
Mario Gundić, ${ }^{1}$ Antonija Tomić, ${ }^{2}$ Rebecca C. Wade, ${ }^{3,4,5}$ Mihaela Matovina, ${ }^{2}$ Zrinka Karačić, ${ }^{2}$ Saša Kazazić, ${ }^{6}$ Sanja Tomićc,*
}

1 Department of Physics, Faculty of Science, University of Zagreb, Bijenička cesta 32, HR-10000 Zagreb, Croatia

2 Division of Organic Chemistry and Biochemistry, Ruđer Bošković Institute, Bijenička cesta 54, HR-10002 Zagreb, Croatia

${ }^{3}$ Molecular and Cellular Modeling Group, Heidelberg Institute for Theoretical Studies (HITS), Heidelberg, Germany

${ }^{4}$ Center for Molecular Biology, DKFZ-ZMBH Alliance, Heidelberg University, Heidelberg, Germany

5 Interdisciplinary Center for Scientific Computing (IWR), Heidelberg University, Heidelberg, Germany

6 Division of Physical Chemistry, Ruđer Bošković Institute, Bijenička cesta 54, HR-10002 Zagreb, Croatia

* Corresponding author's e-mail address: sanja.tomic@irb.hr

RECEIVED: May 23, 2016 * REVISED: July 18, 2016 * ACCEPTED: July 21, 2016

\begin{abstract}
Kelch-like ECH associated protein 1 (Keap1) is a cellular sensor for oxidative stress and a negative regulator of the transcription factor Nrf2. Keap1 and Nrf2 control expression of nearly 500 genes with diverse cytoprotective functions and the Nrf2-Keap1 signaling pathway is a major regulator of cytoprotective responses to oxidative and electrophilic stress. It was found that the metallopeptidase dipeptidyl peptidase III (DPP III) contributes to Nrf2 activation by binding to Keap1, probably by binding to the Kelch domain, and thereby influences Nrf2 activity in cancer. We here first determined that the $K_{D}$ of the DPP III-Kelch domain complex is in the submicromolar range. In order to elucidate the molecular details of the DPP III - Kelch interaction we then built models of the complex between human DPP III and the Keap1 Kelch domain and performed coarse-grained and atomistic simulations of the complexes. In the most stable complexes, the ETGE motif in the DPP III flexible loop binds near the central pore of the six-blade $\beta$-propeller Kelch domain. According to the preliminary HD exchange experiments DPP III binds to the more unstructured end of Kelch domain. According to the results of MD simulations DPP III binding to the Kelch domain does not influence the overall DPP III structure or the long-range domain fluctuations. We can conclude that DPP III forms the stable complexes with the Keap1 Kelch domain by inserting the flexible loop into the entrance to the central pore of the six blade $\beta$-propeller Kelch domain at its more unstructured, N-terminus.
\end{abstract}

Keywords: 'protein-protein interaction', dipeptidyl peptidase III, docking, molecular dynamics, Keap1, microscale thermophoresis (MST).

\section{INTRODUCTION}

$\mathbf{T}$ HE Nrf2-Keap1 signaling pathway is a major regulator of cytoprotective responses to oxidative and electrophilic stress. Nrf2 controls expression of about 200 genes that collectively function to maintain a healthy intracellular reduction-oxidation balance, clear electrophilic xenobiotics and degrade damaged and misfolded proteins. ${ }^{[1]}$ Together, Keap1, a substrate adaptor protein for the Cullin3/Rbx1 ubiquitin ligase, and Nrf2 control the expression of nearly 500 genes with diverse cytoprotective functions. ${ }^{[1]}$ A low concentration of Nrf2 in the basal state is maintained by ubiquitin-dependent proteosomal degradation. Normally, Keap1 binds to Nrf2 and continuously targets Nrf2 for degradation, but loses this ability in response to electrophiles and oxidants. Consequently, Nrf2 accumulates and activates transcription of its downstream target genes.

The transcription factor Nrf2 binds as a monomer to homodimeric Keap1 via two motifs, the DLG and the ETGE motif, whereby the affinity for the ETGE motif is 200-fold greater than for the DLG motif. ${ }^{[1-3]}$ Using FLIM-FRET, Baird and coworkers ${ }^{[1]}$ revealed that the Keap1 - Nrf2 complex exists in two distinct conformations, "open conformation" and "closed conformation." In the basal state, newly translated Nrf2 binds to one subunit of the free Keap1 dimer through its high affinity ETGE motif to form the "open conformation." Subsequently, the low affinity DLG motif of Nrf2 binds to the other subunit of the Keap1 dimer to form 


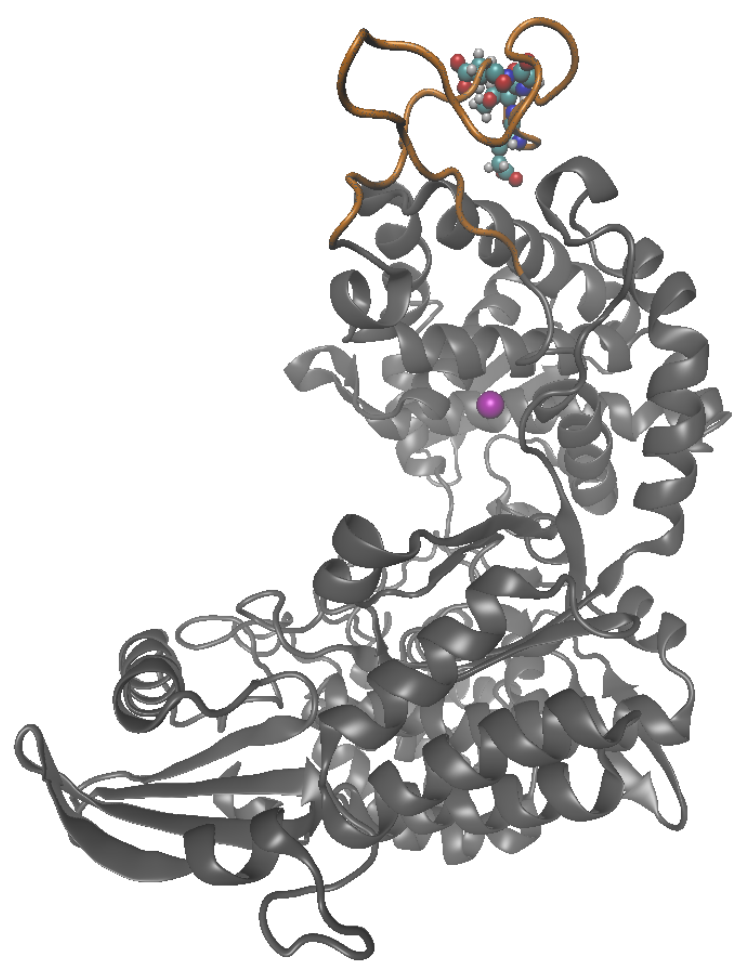

Figure 1. Cartoon representation of the human DPP III 3D structure (PDB code 3FVY). The ETGE motif on the flexible loop (colored orange) of the 'upper', catalytic domain is shown in ball and stick representation, while the zinc ion is represented as magenta sphere.

the "closed conformation." Once the two-site binding is achieved, the lysine residues in the $\alpha$-helix between the DLG and ETGE motifs are in the correct orientation to be ubiquitinated by the Keap1-dependent E3-ligase. ${ }^{[1-3]}$

It was found that proteins containing either an ENGE or ETGE motif like the oncoprotein prothymosin $\alpha^{\left[{ }^{[4]}\right.}$ p62 and DPP III[5] compete with Nrf2 for binding to Keap1, suggesting that this may be a common non-canonical mechanism by which cytoprotective gene expression can be regulated. ${ }^{[6]}$

Dipeptidyl peptidase III (DPP III, EC 3.4.14.4) is a zinc metallopeptidase with relatively broad specificity that sequentially cleaves off dipeptides from unsubstituted amino-termini of 3 to 10 residue long peptides in vitro, showing broad specificity for 4 to 8 residue substrates. ${ }^{[7]}$ It is the sole member of the M49 metallopeptidase family, with a unique active site motif, HEXXXH, and it is the only metalloenzyme among dipeptidyl peptidases. ${ }^{[8,9]}$ DPP III is considered to be a cytosolic enzyme. However, there are reports showing its association with membranes, and most recently its nuclear localization was observed as well..[10]
Extensive investigations of the biochemical properties, structure and dynamics of this protein have been conducted by our group. ${ }^{[11-16]}$ An enhanced level of human DPP III activity and protein was found in endometrial carcinomas and in ovarian malignant neoplasms. ${ }^{[17]}$ Moreover, a correlation between DPP III activity and the aggressiveness of ovarian primary carcinomas was shown. ${ }^{[18]}$ Besides, DPP III, with several other endogenous peptidases in the lens, plays an important role in cataractogenesis by inducing lens opacification through the intracellular turnover of lens proteins. ${ }^{[19]}$

The experiments at cellular level[20] suggested that DPP III binds to the Kelch domain of Keap1 via an ETGE motif and in this way prevents formation of the Keap1NRF2 complex and, consequently, drives the NRF2-dependent transcription. The ETGE motif of DPP III resides in a loop, on the surface of the catalytic domain (see Figure 1), and we found that it is the most flexible part of the human DPP III. ${ }^{[12]}$

Besides in human, this loop was identified in DPP IIIs found in animals and fungi, as well. However, DPP IIIs from plant and bacteria lack this loop (see Supplemental materials).

The motivation for our work was to find out what is the affinity of the DPP III-Kelch interactions and to elucidate molecular details of the interaction. The interaction of DPP III and Kelch domain was assessed experimentally by microscale thermophoresis (MST), a novel technique for the detection and measurement of biomolecular interactions. MST is based on the directed movement of molecules in the temperature gradient that strongly depends on molecular properties such as size, charge, and hydration shell or conformation, and it is sensitive to any change in molecular properties allowing for a precise quantification of molecular interactions. ${ }^{[21]}$ MST measurements with fluorescently labeled Kelch domain and unlabeled DPP III indicate that DPP III and the Kelch domain of Keap1 protein bind with a $\mathrm{Kd}$ in the submicromolar range.

In order to elucidate molecular details of the DPP III - Keap1 Kelch domain interaction, we combined docking with all-atom and coarse grained molecular dynamics simulations. The simulations revealed that presence of the Kelch domain does not influence either the structure of the DPP III domains or the structure of the active site and $\mathrm{Zn}^{2+}$ coordination. The long range domain fluctuations of the two DPP III domains, observed during simulations of human DPP III alone, were also present during the simulations of the DPP III - Keap1 complexes. In the most stable complexes, the ETGE motif on the DPP III flexible loop binds near the central pore of the six blade $\beta$-propeller Kelch domain. 


\section{METHODS}

\section{Protein Expression and Purification}

Recombinant hDPP III with a C-terminal His-tag was expressed from the $\mathrm{DET} 21 \mathrm{~b}$ vector with the hDPP3 gene cloned between the Nhel and Xhol sites.[11] Recombinant Kelch domain of the Keap1 protein (residues 321-609) with an N-terminal His-tag was expressed from the pET15b vector with the Kelch domain cloned between the Ndel and BamHI sites (kind gift from Prof. Mark Hannink, University of Missouri-Columbia, Columbia, MO, USA). ${ }^{[22]}$

Both proteins were overexpressed in $E$. coli strain BL21-CodonPlus (DE3)-RIL +, and purified by Ni-NTA agarose affinity purification as described by Špoljarić et al. (2011). ${ }^{[11]}$ Fractions of high purity (according to the SDSPAGE analysis) were pooled and desalted on PD-10 columns (Amersham Biosciences, Vienna, Austria) equilibrated with $20 \mathrm{mM}$ Tris- $\mathrm{HCl}$ buffer, $\mathrm{pH}=7.4$. Protein concentrations were determined using the protein-dye binding assay, with bovine serum albumin as a standard. ${ }^{[23]}$

\section{Microscale Thermophoresis}

Recombinant Kelch domain was labeled with NT-647 dye using the Monolith NT'M Protein Labeling Kit (amine reactive, NanoTemper Technologies GmbH, Munich, Germany). The concentration of labeled molecule was kept constant at $50 \mathrm{nM}$, while a titration series of 16 dilutions of unlabeled DPP III ( $20 \mu \mathrm{M}$ to $0.6 \mathrm{nM}$ ) in the interaction buffer $(20 \mathrm{mM}$ Tris- $\mathrm{HCl}, \mathrm{pH}=7.4$, $0.05 \%$ Tween) was prepared. Ten microliters of serial dilution of the unlabeled DPP III was mixed with $10 \mu$ of $50 \mathrm{nM}$ fluorescently labeled Kelch domain. Mixed samples were loaded into premium coated glass capillaries and the MST analysis was performed using the Monolith.NT.115 (NanoTemper Technologies $\mathrm{GmbH}$, Munich, Germany). MST measurements were done at $30 \%$ excitation-power and $20 \%$ MST power.

\section{Docking}

The experimentally determined structures of human DPP III, open (oWT) and closed (cWT) forms (PDB codes 3FVY and 3T6B, respectively), and Keap1 Kelch domain (PDB code $1 \mathrm{U6D}$ ), were used as starting structures for docking. For this purpose, the amino acid residues missing in 3FVY, Pro-224-Asp-227, were modeled using the program Modeller9v2 ${ }^{[24]}$ and Ala at position 415 in 3T6B was mutated to Glu to obtain the native sequence. The weak substrate in 3T6B and the crystallographic water molecules were removed. Rigid-body protein-protein docking was performed using two different web servers with default parameters: GRAMM-X (http://vakser.compbio.ku.edu/resources/gramm/grammx) ${ }^{[25]}$ and webSDA (http://mcm.h-its.org/webSDA).[26,27] Two types of complexes were predicted: open and closed, with DPP III in its either open or closed form (PDB codes 3FVY and 3T6B, respectively), respectively, and the Keap1 Kelch domain
(PDB code 1U6D). The best models according to the orientation of the DPP III flexible loop containing ETGE motif and the Keap1 Kelch domain were selected for further simulations. Namely, Hast et al. ${ }^{[20]}$ claimed that DPP III binds to Kelch via an ETGE motif, so we selected the low energy models in which the DPP III flexible loop containing ETGE was closest to the less structured, more narrow, side of the southpaw shaped six-blade $\beta$-propeller Kelch domain (in accord with the results of HD exchange experiment) for further MD simulations.

\section{Simulations.}

All-atom MD simulations

The docked complexes were parametrized using the ff14SB force field ${ }^{[28,29]}$ whereas the $\mathrm{Zn}^{2+}$ parameters from our previous simulations were used. ${ }^{[12]}$ The zinc ion was described with noncovalent parameters only (charge $+2.0 e$, and parameters for van der Waals interactions: raduis $1.22 \AA$, and energy minima of $0.250 \mathrm{kcal} \mathrm{mol}^{-1}$ ).

$\mathrm{Arg}$ and Lys residues were positively charged $(+1 \mathrm{e})$, and all Glu and Asp residues were negatively charged $(-1 e)$, as expected under physiological conditions. The protonation of His residues was checked according to their ability to form hydrogen bonds with adjacent amino acid residues. The prepared complexes were neutralized $\left(29 \mathrm{Na}^{+}\right.$were added) and solvated in a cubic box using TIP3P water molecules and periodic boundary conditions (details of the system preparation could be find in our earlier publication). [12]

Geometry optimized structures were equilibrated for $100 \mathrm{ps}$ ( $30 \mathrm{ps}$ of temperature +70 ps of density adjustments were performed using a $1 \mathrm{fs}$ time step and $N V T$ and $N p T$ conditions, respectively). Followed the productive $N p T$ $\mathrm{MD}$ simulations at $300 \mathrm{~K}$ with the time step of $2 \mathrm{fs}$. The temperature was held constant using Langevin dynamics with a collision frequency of $1 \mathrm{ps}^{-1}$. Pressure was regulated by a Berendsen barostat. Bonds involving hydrogen atoms were constrained using the SHAKE algorithm. The electrostatic interactions were calculated using the particle-mesh Ewald $\operatorname{method}^{[30,31]}$ Simulations were performed within the program package AMBER14 (http://ambermd.org). ${ }^{[32]}$

\section{Steered MD Simulations}

In the crystal structure of human DPP III (PDB code 3FVY), the first Glu (Glu480) from the ETGE motif strongly interacts with two arginines (Arg623 and Arg624) from the neighboring helix in DPPIII, and these interactions remained preserved even after the 100-ns-long MD simulations of closed human DPP III, see Supplemental Figure S1. Since the results of HD exchange showed that in the DPP III - Kelch complexes this loop become much more solvent exposed than in the free enzyme we used the program PyMOL (www.pymol.org) followed by the steered MD simulations to reorient this glutamate towards the partner protein. For this purpose, the Glu480 carboxyl group was weakly pulled, 
with a force constant of $10 \mathrm{kcal} \mathrm{mol}^{-1} \AA^{-2}$, towards the Kelch arginines (Arg415 and Arg483) close to the flexible loop over a period of $100 \mathrm{ps}$. All other simulation conditions were the same as those during the production phase of the MD simulations.

\section{Coarse Grained (cg) MD Simulations}

The initial coarse grained $(\mathrm{cg})$ models of complexes were built in GROMACS 5.07 (www.gromacs.org) using open and closed complexes derived from docking predictions as starting structures. A variant of the Martini force field, EINeDyn(v2), ${ }^{[33]}$ was used, which utilizes a global elastic network between the backbone beads to preserve the secondary structure and uses the positions of $\mathrm{C} \alpha$-atoms as the location of the backbone beads. First we performed several "test calculations" using several different elastic bonds strengths with combined cut-off values of 0.7 and $0.9 \mathrm{~nm}$. Since the elastic bond strength of $500 \mathrm{~kJ} \mathrm{~mol}^{-1} \mathrm{~nm}^{-2}$ and a cutoff of $0.7 \mathrm{~nm}$ ensured the conservation of secondary structure and the mobility of the tertiary structures during the simulations, these parameters were used in subsequent runs.

The cg structures were minimized in vacuum, solvated in a cubic box, filled with water particles (using a box of pure water solvent, equilibrated at $\mathrm{T}=300 \mathrm{~K}, \mathrm{p}=1 \mathrm{bar}$, http://md.chem.rug.nl/index.php/downloads/exampleapplications/63-pure-water-solvent). The prepared systems were neutralized by adding $\mathrm{Na}^{+}$ions and minimized using the steepest descent algorithm. Simulations were performed using the leap-frog algorithm with an $\mathrm{NpT}$ ensemble at $300 \mathrm{~K}$ and 1 bar with a time step of $10 \mathrm{fs}$. Electrostatic interactions were calculated using a Verlet cut-off scheme, with each simulation starting with the same seed number. The temperature coupling was done using V-scale (modified Berendsen thermostat) and the pressure was held constant (with reference pressure of 1 bar) using the Parrinello-Rahman barostat (with semi uniform scaling of box vectors, coupling time constant $\tau$ _ $p=12.0 \mathrm{ps}$ and compressibility of $\left.3 \times 10^{-4} \mathrm{bar}^{-1}\right)$. On average, the systems were simulated for $10 \mu \mathrm{s}$, with the exception of the open complex which was simulated for $44 \mu \mathrm{s}$. Structures and energies were saved every 10000 steps.

\section{Data Analysis}

In order to analyze and characterize the interactions between DPP III and the Kelch domain during simulations, as well as to determine possible changes in their structure and the most relevant protein motions, several geometric parameters were analyzed with the program ptraj, an AMBER14 module. ${ }^{[34]}$ Besides a set of pairwise distances, the backbone atom root-mean squared deviation (RMSD), the $\mathrm{C} \alpha$ atom root-mean squared fluctuations (RMSF) and the radius of gyration (Rgyr) (Supplemental equations 1-3, respectively) were monitored during the simulation. The distances between D186-S500 and Q400-S500 C $\alpha$ atoms, $d_{1}$ and $d_{2}$, respectively, were also computed (see Supp. Figure S2). Our earlier studies on DPP III have shown that these distances appropriately describe the mutual orientation of the two domains. Besides analysis of the geometric parameters the binding free energies were calculated using MM-GBSA (Molecular Mechanics Generalized Born Surface Area) and MM-PBSA (Molecular Mechanics Poisson Boltzman Surface Area) methods, as well. Further on, the residue-based relative free energies were calculated, using the MM-GBSA approach, to highlight the residues relevant for stabilization of the complexes with DPP III in either open or closed form. For this purpose, the 1-ns-long trajectories generated during the last stage of MD simulations were considered. The calculations were performed for the enzyme with a relative dielectric constant of 2.0 immersed in solvent with a relative dielectric constant of 80 . Structures were sampled every 20 ps.

\section{RESULTS AND DISCUSSION}

\section{Microscale Thermophoresis}

The interaction between DPP III and the Kelch domain of Keap1 protein was assessed by MST. The thermophoretic movement of the fluorescently labeled Kelch domain in the presence of DPP III as titrant was measured by monitoring the fluorescence distribution $F$ inside the capillary (Figure 2). Formation of complex between DPP III and Kelch domain of Keap1 protein was observed at submicromolar concentrations of the titrant. The $K_{D}$ of $661 \pm 87 \mathrm{nM}$ for the DPP III-Kelch complex was determined from the binding curve.

\section{Docking}

For the open form of DPP III, the GRAMM-X server predicted two significantly different DPP III - Keap1 Kelch complexes, see Figure 3 . In one, DPP III is bound to the more

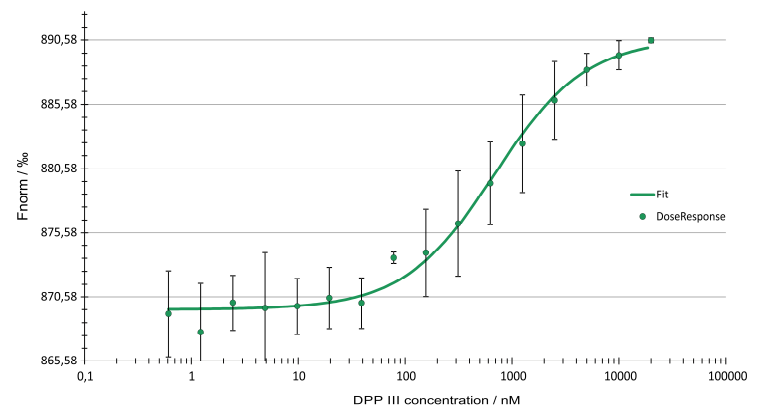

Figure 2. Analysis of DPP III-Kelch interaction by MST. In the MST experiments, the concentration of labeled Kelch was kept constant at $50 \mathrm{nM}$, while the concentration of the unlabeled DPP III was varied $(0.6 \mathrm{nM}-20 \mu \mathrm{M})$. The binding curve was obtained from two independent measurements at $30 \%$ excitation-power and $20 \%$ MST power. 

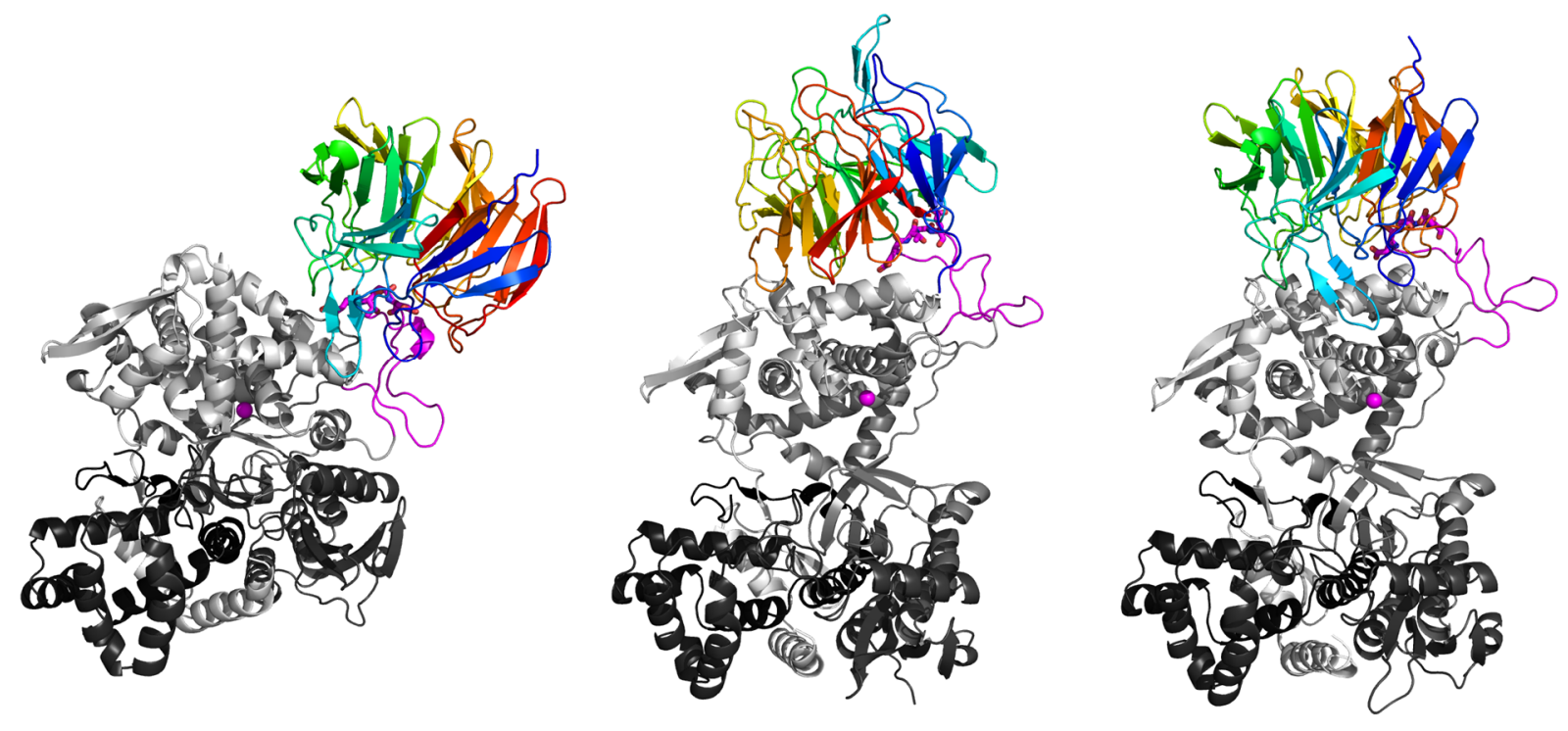

Figure 3. Cartoon representations of GRAMM-X docking predictions for DPP III - Keap1 Kelch complexes. The ETGE motif, represented by sticks, and the flexible loop, are colored magenta. Orientations are shown for Keap1 Kelch with respect to the closed form DPP III (GX-DPP III-closed, left), and two variants for the open form of DPP III - Keap1 Kelch complexes, with opposite orientation of Keap1 Kelch (GX'-DPP III-open, middle), and the same orientation of Keap1 Kelch as for the closed form DPP III (GX-DPP III-open, right). DPP III and Kelch are coloured from N to C terminus in a spectrum ranging from black to white and from blue to red, respectively. The zinc ion is shown as a magenta sphere.
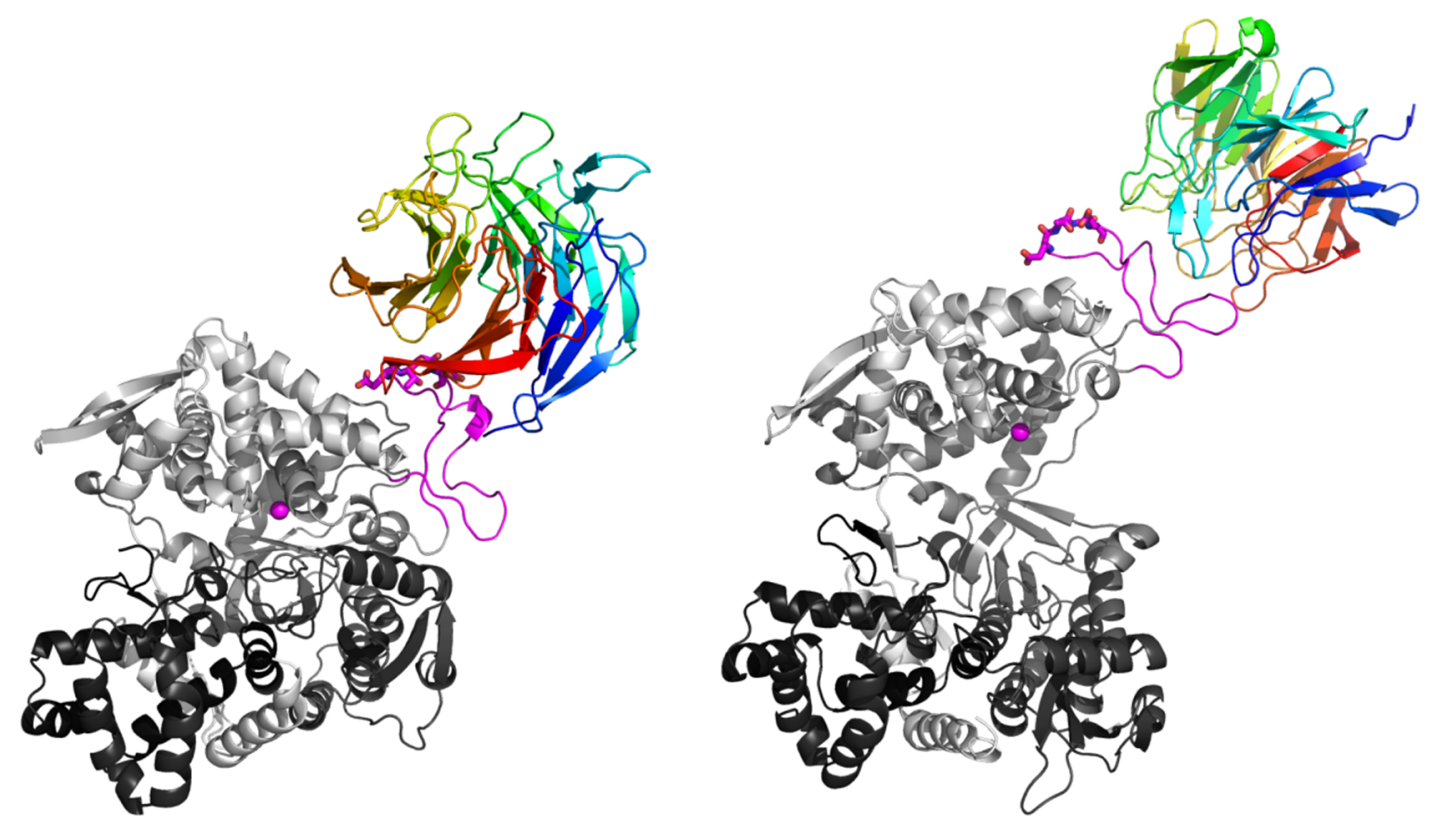

Figure 4. Cartoon representation of the DPP III - Keap1 Kelch encounter complexes predicted with the webSDA server. The ETGE motif, represented by sticks, and the flexible loop, are colored magenta. The encounter complex predicted for the closed DPP III form is given on the left (SDA'-DPP III-closed), and for the open DPP III form on the right (SDA-DPP III-open). The DPP III and Keap1Kelch domain are colored from $\mathrm{N}$ to $\mathrm{C}$ terminus in a spectrum ranging from black to white and from blue to red, respectively. The zinc ion is shown as a magenta sphere. 
unstructured end of Kelch (Figure 3, right, GX-DPP III-open complex) and in the other to the more structured, the Kelch domain C-terminus (Figure 3, middle, GX'-DPP III-open complex). For the closed form of DPP III, the GRAMM-X server, as expected, predicted binding of the DPP III flexible loop to the more unstructured end of Kelch (Figure 3, left, GX-DPP III-closed complex). Using the webSDA server (Figure 4) complexes similar to those predicted by GRAMM-X were obtained for the open form of DPP III (SDA-DPP IIIopen complex). For the complex with the closed form of DPP III, the opposite orientation of Keap1 Kelch and DPP III was found with respect to the one predicted by GRAMM-X (SDA'-DPP III-closed complex).

\section{All-atom MD Simulations.}

Almost 600 ns of productive, all-atom MD simulations were performed for the human DPP III - Kelch complexes (see Supplemental Table S1). Most of these simulations (about $440 \mathrm{~ns})$ were performed for the DPP III - Kelch complex with DPP III initially in the open form, and about $200 \mathrm{~ns}$ (120 ns $+80 \mathrm{~ns}$ ) for the DPP III - Kelch complex with DPP III in the closed form, of that 120 ns for the complex with the expected orientation of DPP III and Kelch and 80 with opposite orientation of the Kelch domain, i.e. Kelch is interacting with human DPP III with its more structured, wider side of the southpaw shaped Kelch domain instead with its less structured more narrow side (see the last section of Supplemental Info). Since the results of preliminary HD exchange experiment predicted binding of DPP III to the more unstructured, N-terminal part of the Kelch domain (for details see Supplemental Info), in this article we concentrated to the results of the simulations starting from the complexes in which this condition was satisfied.

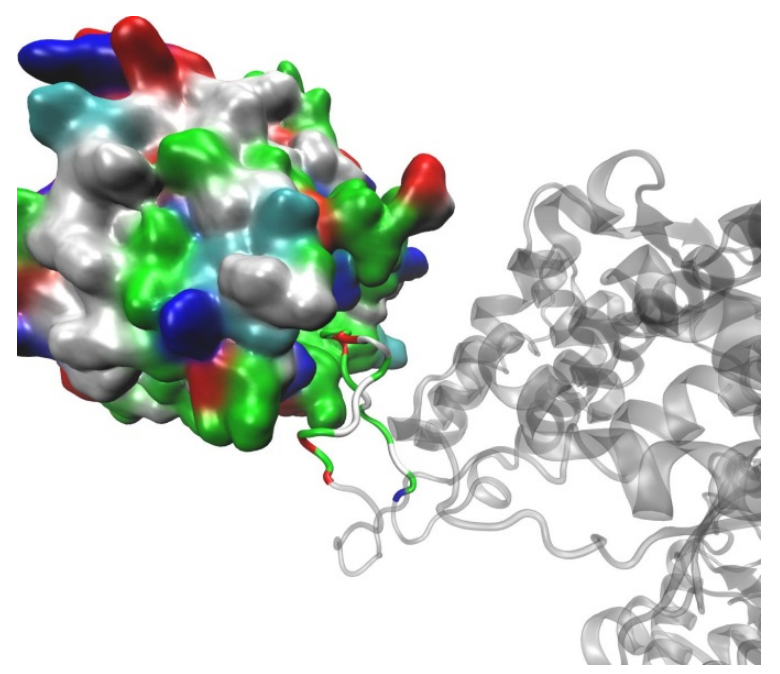

\section{All-atom MD Simulations with DPP III in its Open Form}

The complex obtained using the webSDA webserver, with DPP III bound on the same side as the NRF2 peptide (see for example crystal structure with the PDB code 2FLU) (Figure 4 right), complex named SDA-DPP III-open, was simulated for about $200 \mathrm{~ns}$. During the simulations, the DPP III flexible loop with the ETGE motif accommodated near the central pore of the six blade $\beta$-propeller Kelch domain (Figure 5). Neither the Kelch domain structure nor the structure of the DPP III domains changed significantly during the simulations (the RMSDs remained below $2 \AA$ during the simulation, see Supp. Figure S3). The coordination of the zinc ion also remained preserved during the simulations (Supp. Figure S4). The metal cation, $\mathrm{Zn}^{2+}$, was coordinated by histidines $\mathrm{H} 450$ and $\mathrm{H} 455$ and glutamates E451 and E508 during simulations. E451 coordinated the zinc ion monodentately (first 150 ns with OE1, and the last 50 ns with OE2) while the coordination by E508 was interchangeably, monodentate and bidentate (Supp. Figure S4). During the simulations, the long range domain fluctuations resulted in the partial closure of the protein, i.e. during the simulations Rgyr decreased from ca. 28 to $26.5 \AA$ (Supp. Figure S5), and distances $\mathrm{d} 1$ and $\mathrm{d} 2$, between D186-S500 and Q400-S500 $\mathrm{C} \alpha$ atoms, significantly changed during the simulations (Figure S6). Such long range fluctuations have been determined during simulations of DPP III alone, as well (Figure S7).

It should be noted that the RMSD of the flexible loop (black line in Figure S3) is significantly lower than the RMSD in simulations of DPP III alone (green line in Figure S8, top). During the simulations, Glu483, the second Glu of the ETGE motif, established strong hydrogen bonds and electrostatic interactions with charged and polar Kelch residues, Arg415

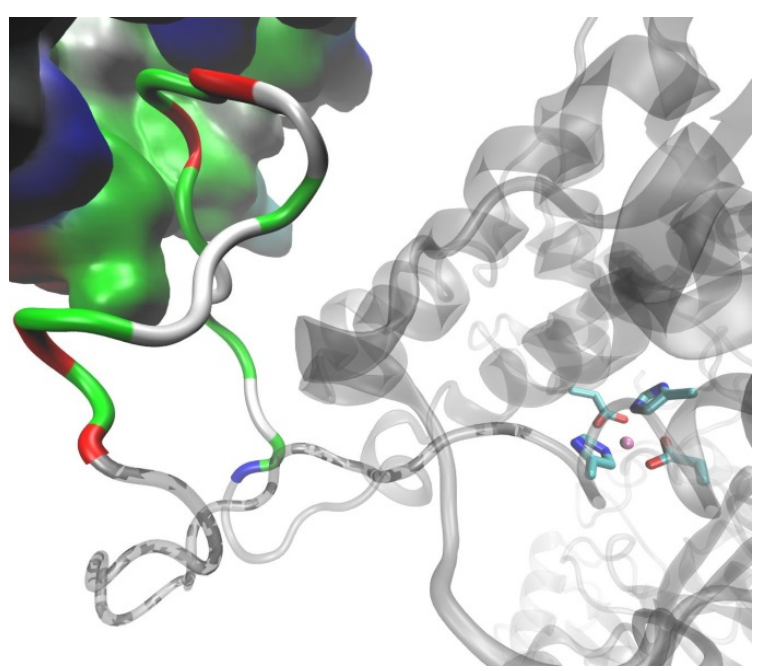

Figure 5. h.DPP III - Kelch interactions. DPP III is shown as a gray ribbon, except for the flexible loop (with the ETGE motif, residue numbers 480 to 483 ) which is colored according to the residue type. The Keap1 Kelch domain is represented as a surface colored by residue type. The zinc ion is shown as a magenta sphere, and its ligands as sticks. 


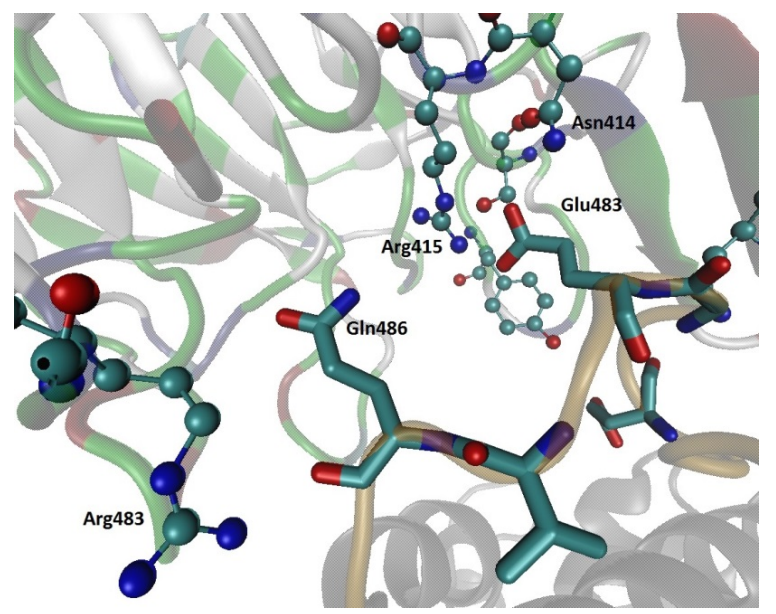

Figure 6. h.DPP III - Kelch interactions in the open DPP III Kelch domain complex after 200 ns of MD simulations. Initial complex was determined with the webSDA web server. Amino acid residues from the ETGE motif and DPP III Asn486 are shown in stick representation, and the residues from the Kelch domain that they interact with are shown as ball and stick. DPP III is shown as gray ribbon, except the flexible loop that is colored orange. The Kelch domain is colored according to the residue type.

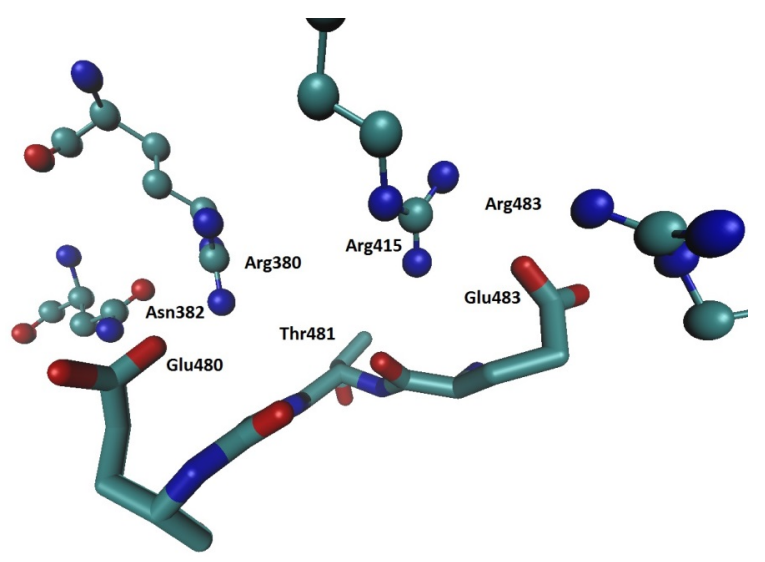

Figure 7. h.DPP III - Kelch interactions at the end of 100 ps of restrained MD simulations. Amino acid residues from the ETGE motif are shown in stick, and the residues from the Kelch domain with which they interact with are shown as ball and stick.

and Asn414 and Ser363, respectively. GIn486 established a strong H-bond with Kelch Arge483 and Phe478. The first Glu of the ETGE motif interacts with Tyr334 and with Asn387 from the Kelch domain (Figure 6).

In the crystal structure of the Kelch domain - NRF2 peptide complex (PDB code 2FLU), the orientation of the ETGE motif is somewhat different from that determined by
MD simulations of the complexes predicted by the docking web servers, webSDA and GRAMM-X. In order to adjust (reorient) the DPP III ETGE motif to be as similar as possible to the NRF2 peptide one, we either modified the loop position manually using the program Pymol or used the weakly restrained MD simulations. During 100 ps of the restrained simulations, the DPP III ETGE motif adopted an orientation close to the orientation of the NRF2 peptide, complex named SDA-DPP III-openSt-1, see Table S1). The first and the last glutamate (Glu480 and Glu483, respectively) of the ETGE motif interacted with two Arg residues of the Kelch domain, R415 and R380, respectively. Gly482 interacted with Ser602 and Thr481 with Ala556 and Tyr572 (not shown). However, such an orientation of the loop was not stable and during the following $60 \mathrm{~ns}$ of unrestrained simulations, Kelch Arg483 moved away from DPP III (Figure S7). As in the $200 \mathrm{~ns}$ long simulation described above, the structures of the DPP III domains and the Kelch did not changed significantly and the coordination of the $\mathrm{Zn}^{2+}$ was preserved.

All-atom MD Simulations with DPP III in its Closed Form During 120 ns of MD simulations of the complex obtained using the Gramm-X docking server, with DPP III in its closed form (Figure 3 left), complex named GX-DPP III-closed, the structure of the interacting proteins Kelch domain and DPP III did not changed significantly (the RMSDs remained below $2 \AA$ during the simulation). The interactions between the DPP III and Kelch domain established during the simulations are shown in Figure 8.

The zinc ion was six-coordinated during the entire simulation of $120 \mathrm{~ns}$. The coordinating residues are His450, His455, Glu451 and Glu508 (Supp. Figure S10), wherein both glutamates coordinate $\mathrm{Zn}^{2+}$ bidentately (not shown). The RMSD of each DPP III domain as well as of the Kelch domain remained below $1.5 \AA$, and the RMSD of the flexible loop was about $1 \AA$ (Supp. Figure S11). During the simulations, DPP III remained closed (Rgyr about $25.5 \AA ̊$ ). In order to adjust (reorient) the DPP III ETGE motif to be as similar as possible to the NRF2 peptide orientation in the crystal structure, we modified the loop position manually using program PYMOL. However, during 80 ns of MD simulation, the DPP III flexible loop moved to the edge of the six-blade Kelch domain and interactions between Glu483 (ETGE motif) and the Kelch residues Arg459 and His436 were established (Figure S9).

\section{MM-GBSA and MM-PBSA binding free energies}

From the binding free energies calculated for the complexes with the DPP III structure initially in its open and closed form, as well as from the residues based free energy difference plot (see Supplemental Table S2 and Figure S12) it is obvious that the complex with the closed DPP III structure is more stable than the complex with the open one. This difference is a result of a number of the intermolecular 


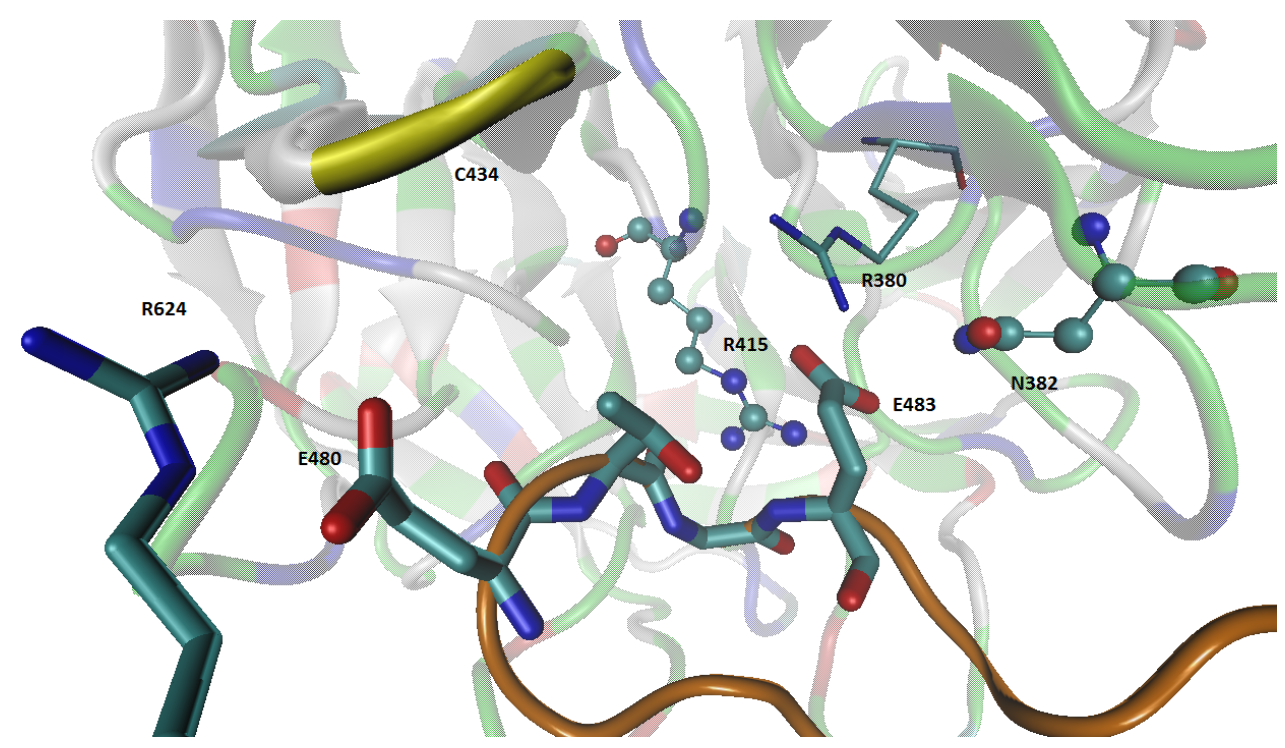

Figure 8. The DPP III - Kelch interactions. Amino acid residues from the ETGE motif and DPP III Arg624 are shown as sticks, and the residues from the Kelch domain that interact with DPP III are shown as ball and sticks (Asn382, Arg415) except Arg380 shown also in stick representation. DPP III is given as gray ribbon, except the flexible loop (orange), and Kelch as a ribbon colored according to the residue type. Glu483 (ETGE) besides with the Kelch residues Arg380, Asn382, Arg415, established the strong electrostatic interaction with the residue from the Kelch N terminus, Ser363 (not shown).

protein-protein interactions that are tiny more stabilizing in the complex with DPP III in its closed form than in the complex with open DPP III. Among them, the interaction between DPP III Gln483 and Kelch Gln337, which is in the 'closed complex' in the form of double $\mathrm{H}$-bond, is the most relevant. In the open complex these two residues are more distant and differently oriented.

\section{Coarse Grained (CG) MD Simulations}

Three types of complexes were simulated: "open" and closed complexes predicted by web-SDA (Figure 4 right and left, respectively) and closed complex predicted by Gramm$X$ server (Figure 3 left).

\section{Open complex (web-SDA variant)}

The structure predicted by webSDA was chosen as the starting structure for building a coarse grain version of the complex with the open form of DPP III. The system was simulated for $44 \mu$ s during which we traced noticeable movement of DPP III domains resulting in a significant increase of the protein compactness (see Figures 9, 10 and S13). The interactions between the DPP III and Kelch stabilized after about $22 \mu$ s as can be concluded from the RMSD plot (Figure S14). Accordingly, the distances between glutamates from the ETGE motif and Kelch domain (Glu483 ${ }^{\text {DPP3}}-$ Ser363 Kelch, Glu483DPP3-Arg380 Kelch, Glu483 DPP3-Asn382 ${ }^{\text {Kelch }}$, Glu480 DPP3-Arg415 ${ }^{\text {Kelch }}$, Glu480 8 DP3_ Ser508 Kelch, Glu480DPP3-Tyr525Kelch) did not change significantly during the last $22 \mu \mathrm{s}$ of CG MD simulations (Figure S15).
According to RMSF, binding of Kelch domain does not influence significantly the local flexibility of DPP III (see Figure S16 in comparison with RMSF profile given in references 13 and 17). The binding free energies calculated for several different DPP III - Kelch complexes, the starting, middle and final structure, generated during $44 \mu$ s long CG MD simulation are given in Table 1.

\section{Closed Complex (Gramm-X Variant)}

Coarse grained model of the complex with closed form DPP III as predicted by Gramm-X server was simulated for $10 \mu \mathrm{s}$ (see Figure 11). During the simulation, distance $\mathrm{d} 1$ slightly

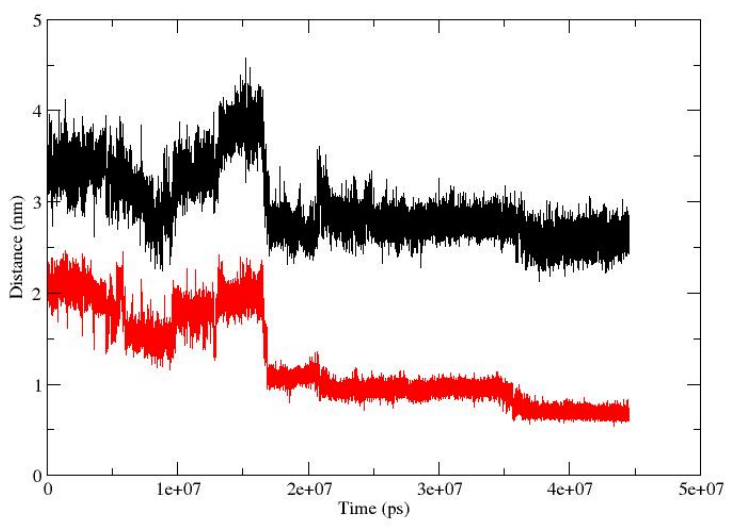

Figure 9. Distances d1 (black) and d2 (red) traced during 44 $\mu$ s of CG simulation of the DPP III - Kelch complex, with initial DPP III in its open form. 

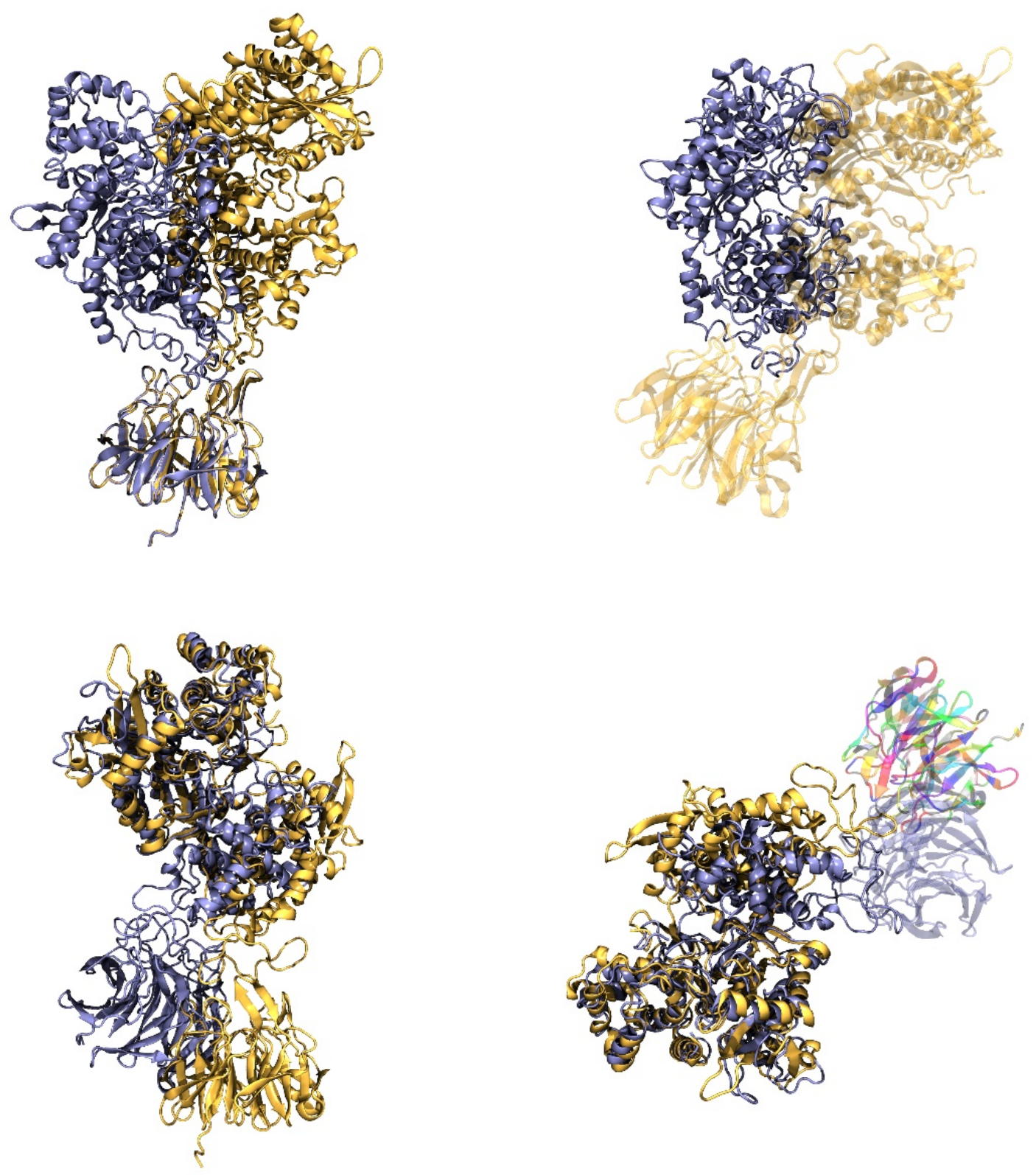

Figure 10. Overlaid, back to all atom representation mapped, structures of the starting and final conformations of the DPP III (open) - Keap1 Kelch complex simulated for 44 ss using the CG MD simulation. Starting structures are colored yellow and the final ones are colored blue. Overlay was done using Keap1 (top) and hDPP III as a reference structure (bottom). In the lower right Figure the Kelch domain from the structure of the initial complex (after $44 \mu \mathrm{s}$ of CG MD simulations) is colored by residue.

decreased while distance $d 2$ increased (Figure S17). Overall, the compactness of DPP III slightly increased (Figure S18), i.e. radius of gyration changed less than $0.5 \AA$. After about 3 $\mu \mathrm{s}$, interactions between DPP III and Kelch stabilized and the mean intermolecular, DPP III - Kelch, distances between the ETGE motif and Kelch residues (Glu483 ${ }^{\text {DPP3}}-$ Ser363 ${ }^{\text {Kelch, }}$

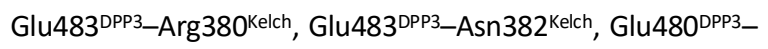

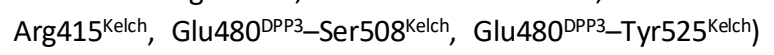

remained unchanged for the rest of the simulation (Figures S19 and S20). After about $7 \mu \mathrm{s}$, the orientation of the DPP III domains changed, and the protein compactness further increased (Figure S18). The binding free energies calculated for several different DPP III - Kelch complexes generated during $10 \mu$ s long CG MD simulation are given in Table 2. As in the case of the simulations of the GRAMMX (DPP III closed) complex, the complex stability increased during the CG MD simulations. 
Table 1. The average binding free energies for calculated for the starting, middle and the final structure of the $44 \mu$ s long simulation of the DPP III - Kelch complex with DPP III initially in its open form (SDA variant).

\begin{tabular}{cc}
\hline Simulation time / ps & $\begin{array}{c}\text { The average binding free energy of the } \\
\text { DPP III - Kelch complexation (in kJ/mol ) }\end{array}$ \\
\hline 1,00E+00 & -4.6 \\
$1,00 E+05$ & -10.5 \\
$4,40 E+07$ & -38.2 \\
\hline
\end{tabular}
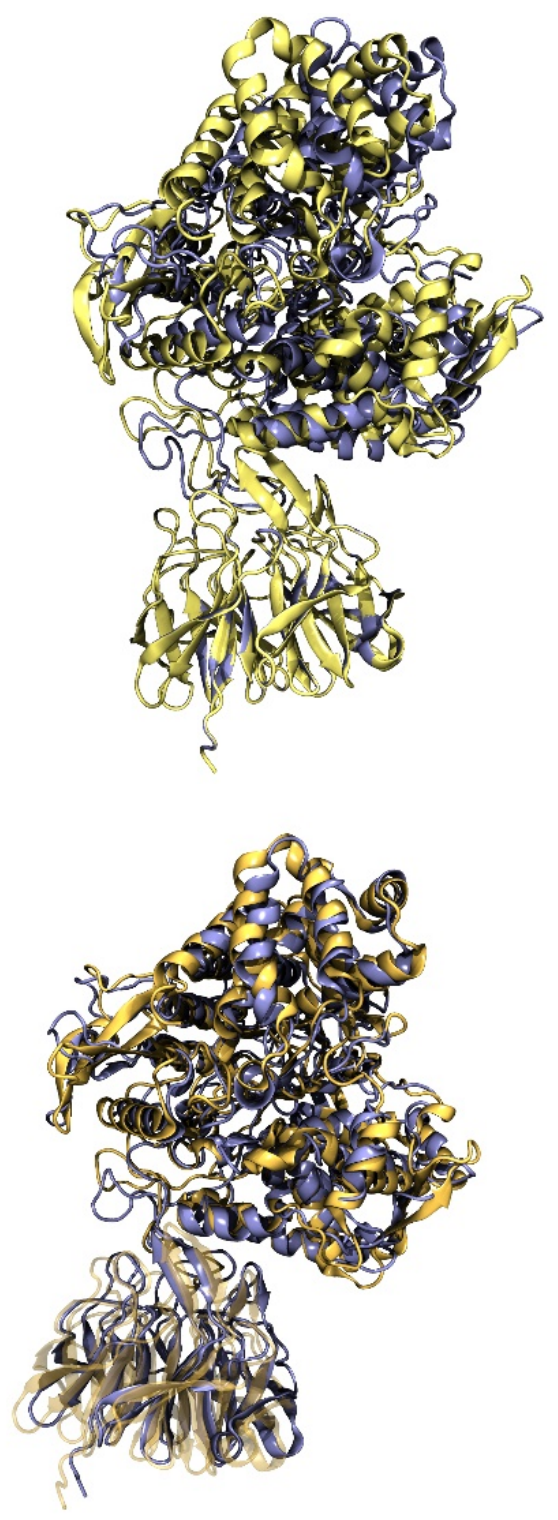

Table 2. The average binding free energies for calculated for three different conformations of the DPP III - Kelch complex generated during the $10 \mu \mathrm{s}$ long CG MD simulation of the complex with DPP III initially in its closed form (Gramm-X variant)

\begin{tabular}{cc}
\hline Simulation time / ps & $\begin{array}{c}\text { The average binding free energy of the } \\
\text { DPP III - Kelch complex (in kJ mol-1) }\end{array}$ \\
\hline $1,00 \mathrm{E}+00$ & -2.3 \\
$1,00 \mathrm{E}+06$ & -20.2 \\
$1,00 \mathrm{E}+07$ & -28.5 \\
\hline
\end{tabular}
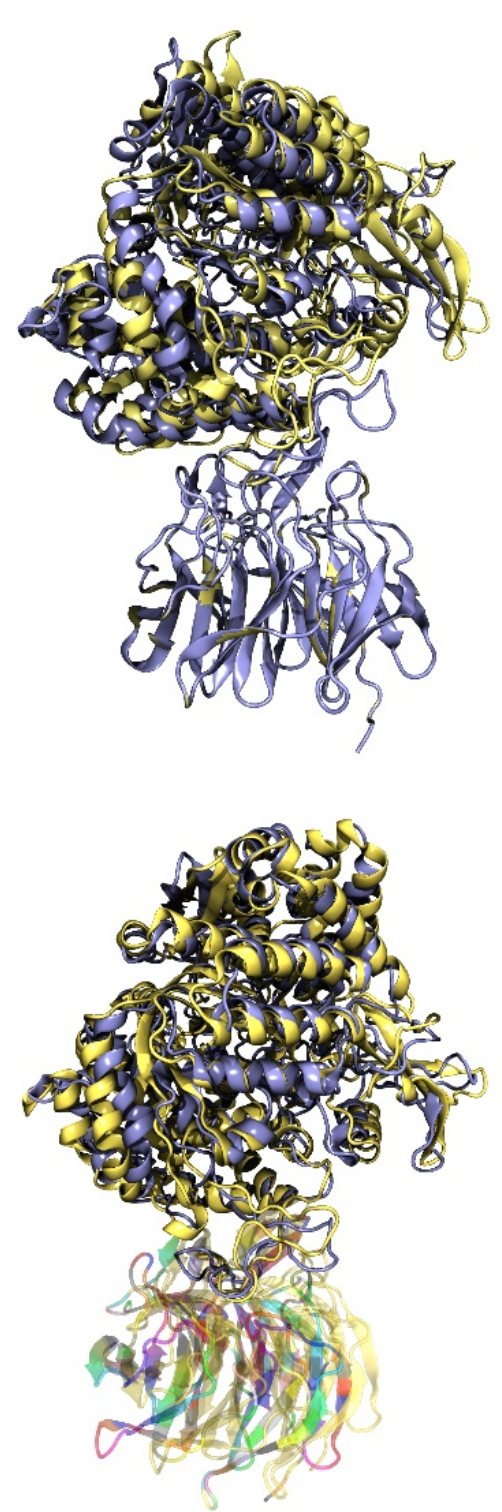

Figure 11. Overlaid, back to all atom representation mapped, structures of the starting and final conformations of the DPP III (closed) - Keap1 Kelch complex simulated for $10 \mu$ s using the CG MD simulation. Starting structures are colored yellow and the final ones are colored blue. Overlay was done using Keap1 (top) and DPP III as a reference structure (bottom). In the lower right Figure the Kelch domain from in the structure of the final complex (after $10 \mu \mathrm{s}$ of CG MD simulations) is colored by residue. 


\section{Closed Complex (webSDA Variant)}

The orientation of DPP III and Kelch in this complex significantly differs from the orientation in the previously described complexes. In this complex, DPP III interacts with the more structured, wider end of the southpaw shaped Kelch domain. However, the complex remained stable and its overall conformation hardly changed during the simulation time $(10 \mu \mathrm{s})$, see Figures S21 and S22. Distances $d 1$ and $d 2$ (Asp186-Ser500 and GIn400-Ser500) slightly increased, but the overall compactness of DPP III increased (Figures S23 and S24) during the $10 \mu$ s long simulation. The mode of DPP III - Kelch binding did not influence the local protein fluctuations, i.e. the pattern of amino acid residues fluctuation determined during both SDA and Gramm-X variant closed complex simulations are very similar.

It is interesting that despite different starting structures and different conformation of DPP III in the initial complexes, GRAMMX (DPP III closed), SDA (DPP III open), and SDA (DPP III closed), similar set of DPP III residues takes part in intermolecular, protein-protein interactions in the complexes obtained by CG MD simulations (see Supplement table S3). Regarding the amino acid residues from the Kelch domain similar set of the interacting residues is present in the first two complexes. About $81 \%$ of the interacting residues (either DPP III or Kelch) determined in the final DPP III-Kelch GRAMMX (DPP III closed) complex is also present in the final DPP III-Kelch SDA (DPP III open) complex. However, in the third (SDA, DPP III closed) complex, completely different region of the Kelch domain interacts with DPP III.

\section{CONCLUSION}

For the first time formation of the DPP III - Kelch domain complex was confirmed by MST measurements and the binding affinity, with a $K_{D}$ value of $661 \pm 87 \mathrm{nM}$, was determined.

Docking study predicted several interfaces for DPP III - Kelch binding. In the present study we concentrated on the results of simulations starting from the structures in which the DPP III flexible loop binds to the same site of the Kelch domain as the NRF2 peptide in the crystal structure (PDB code $2 \mathrm{FLU}$ ) and which is in agreement with the results of preliminary $H D$ exchange experiment. According to the results of both, all-atom and coarse-grained MD simulations, we can conclude that binding of the Keap1 Kelch domain does not influence the structure of the DPP III domains, the metal ion coordination, and the local flexibility pattern. The present study clearly showed that DPP III binds to the Kelch domain and indicated their binding interface.
Acknowledgment. This work was supported by the Croatian Science Foundation (Project: "7235 Flexibility, activity and structure correlations in the dipeptidyl peptidase III family", the Croatian National Grid Infrastructure (CRO NGI, http://www.cro-ngi.hr/), the Alexander von Humboldt Foundation (Project: "Study of plant enzymes from metallopeptidase families M20 and M49"), the Klaus Tschira Foundation, and by European Union's Seventh Framework Programme for Research and Technological Development under grant agreement No. 316289 InnoMol, FP7-REGPOT-2012-2013-1.

The expression plasmid pET15b-Kelch was a kind gift from Prof. Mark Hannink, University of Missouri-Columbia, Columbia, MO, USA.

MST measurements were performed on the Monolith.NT.115 instrument at the Division of Biochemistry, Chemistry Department, Faculty of Science, University of Zagreb.

We kindly thank Jasmina Rokov Plavec and Igor Sviben from the Division of Biochemistry, Chemistry Department, Faculty of Science, University of Zagreb for the help with MST measurements, and the interpretation of the results.

Supplementary Information. Supporting information to the paper is enclosed to the electronic version of the article at: http://dx.doi.org/10.5562/cca2916.

\section{REFERENCES}

[1] L. Baird, S. Swift, D. Llères, A. T. Dinkova-Kostova, Biotechnol. Adv. 2014, 32, 1133.

[2] M. McMahon, T. Thomas, K. Itoh, M. Yamamoto, JD. Hayes, J. Biol. Chem. 2006, 281, 24756.

[3] K. I. Tong, A. Kobayashi, F. Katsuoka, M. Yamamoto, Biol. Chem. 2006, 387, 1311.

[4] R. N. Karapetian, A. G. Evstafieva, I. S. Abaeva, N. V. Chichkova, G. S. Filonov, Y. P. Rubtsov et al., Mol. Cell. Biol. 2005, 25, 1089.

[5] Y. Liu, J. T. Kern, J. R. Walker, J. A. Johnson, P. G. Schultz, H. Luesch, Proc. Natl. Acad. Sci. U. S. A. 2007, 104, 5205.

[6] B. E. Hast, D. Goldfarb, K. M. Mulvaney, M. A. Hast, P.F. Siesser, F. Yan F, Cancer Res. 2013, 73, 2199.

[7] M. Abramić, M. Zubanović, Lj. Vitale, Biol. Chem. Hoppe-Seyler 1988, 369, 29.

[8] M. Abramić, J. Špoljarić, Š. Šimaga, Period. Biolog. 2004, 106, 161.

[9] N. D. Rawlings, M. Waller, A. J. Barrett, A. Bateman, Nucleic Acids Res. 2014, 42, D503.

[10] Ž. Mačak Šafranko, S. Sobočanec, A. Šaric, N. Jajčanin-Jozić, Z. Krsnik, G. Aralica, T. Balog, M. Abramić, J. Endocrinol. Invest. 2014, 38, 471. 
[11] J. Špoljarić, A. Tomić, B. Vukelić, B. Salopek-Sondi, D. Agić, S. Tomić, M. Abramić, Croat. Chem. Acta 2011, 84, 259.

[12] A. Tomić, M. González, S. Tomić, J. Chem. Inf. Model. 2012, 52, 1583.

[13] G. A. Bezera, E. Dobrovetsky, R. Viertlmayr, A. Dong, A. Binter, M. Abramić, P. Macheroux, S. DhePaganon, K. Gruber, Proc. Natl. Acad. Sci. U. S. A. 2012, 109, 6525.

[14] A. Tomić, S. Tomić, Dalton Transactions 2014, 43, 15503.

[15] N. Jajčanin Jozić, S. Tomić, M. Abramić, J. Biochem. 2014, 155, 43.

[16] A. Tomić, M. Berynskyy, R. C. Wade, S. Tomić, Mol. BioSyst. 2015, 11, 3068.

[17] Š. Šimaga, D. Babić, M. Osmak, J. llić-Forko, Lj. Vitale, D. Miličić, M. Abramić, Eur. J. Cancer 1998, 34, 399.

[18] Š. Šimaga, D. Babić, M. Osmak, M. Šprem, M. Abramić, Gynecol. Oncol. 2003, 91, 194.

[19] H. Zhang, Y. Yamamoto, S. Shumiya, M. Kunimatsu, K. Nishi, I. Ohkubo, K. Kani, Histochem. J. 2001, 33, 511.

[20] B. E. Hast, E. W. Cloer, D. Goldfarb, H. Li, P.F. Siesser, F. Yan, V. Walter, N. Zheng, D. N. Hayes, M. B. Major, Cancer Res. 2014, 74, 808.

[21] M. Jerabek-Willemsen, T. André, R. Wanner, H. M. Roth, S. Duhr, P. Baaske, D. Breitsprecher, J. Mol. Struct. 2014, 1077, 101.

[22] X. Li, D. Zhang, M. Hannink, L. J. Beamer, Acta Crystallogr., Sect. D: Biol. Crystallogr. 2004, 60, 2346.

[23] M. M. Bradford, Anal. Biochem. 1976, 72, 248.

[24] A. Sali, T. L. Blundell, J. Mol. Biol. 1993, 234, 779.

[25] A. Tovchigrechko, I. A. Vakser, Nucleic Acids Res. 2006, 34, W310.
[26] R. R. Gabdoulline, R. C. Wade, Biophys. J. 1997, 72, 1917.

[27] M. Martinez, N. J. Bruce, J. Romanowska, D. B. Kokh, M. Ozboyaci, X. Yu, M. Öztürk, S. Richter, R. C. Wade, Comput. Chem. 2015, 36, 1631.

[28] K. Lindorff-Larsen, S. Piana, K. Palmo, P. Maragakis, J. L. Klepeis, R. O. Dror, D. E. Shaw, Proteins, 2010, 78, 1950.

[29] J. A. Maier, C. Martinez, K. Kasavajhala, L. Wickstrom, K. E. Hauser, C. Simmerling, J. Chem. Theory Comput. 2015, 11(8), 3696.

[30] T. Darden, D. York, L. Pedersen, J. Chem. Phys. 1993, 98, 10089.

[31] U. Essmann, L. Perera, M. L. Berkowitz, T. Darden, H. Lee, L. G. Pedersen, J. Chem. Phys. 1995, 103, 8577.

[32] D. A. Case, T. E. Cheatham, T. Darden, H. Gohlke, R. Luo, K. M. Merz, A. Onufriev, C. Simmerling, B. Wang, R. J. Woods, J. Comput. Chem. 2005, 26, 1668.

[33] X. Periole, M. Cavalli, S. Marrink, A. Ceruso, J. Chem. Theory Comput. 2009, 5, 2531.

[34] D. A. Case, J. T. Berryman, R. M. Betz, D. S. Cerutti, T. E. Cheatham III, T. A. Darden, R. E. Duke, T. J. Giese, H. Gohlke, A. W. Goetz, N. Homeyer, S. Izadi, P. Janowski, J. Kaus, A. Kovalenko, T. S. Lee, S. LeGrand, P. Li, T. Luchko, R. Luo, B. Madej, K. M. Merz, G. Monard, P. Needham, H. Nguyen, H. T. Nguyen, I. Omelyan, A. Onufriev, D. R. Roe, A. Roitberg, R. Salomon-Ferrer, C. L. Simmerling, W. Smith, J. Swails, R. C. Walker, J. Wang, R. M. Wolf, X. Wu, D. M. York, P. A. Kollman, AMBER 2015, 2015, University of California, San Francisco. 


\section{Human DPP III - Keap1 Interactions: A Combined Experimental And Computational study}

M. Gundić, A. Tomić, R. C. Wade, M. Matovina, Z. Karačić, S. Kazazić and S. Tomić*

\section{SEQUENCE ALIGNMENT}

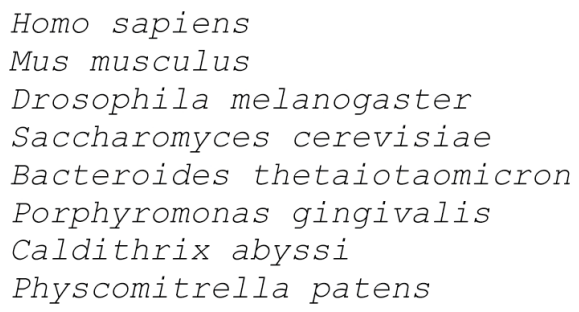

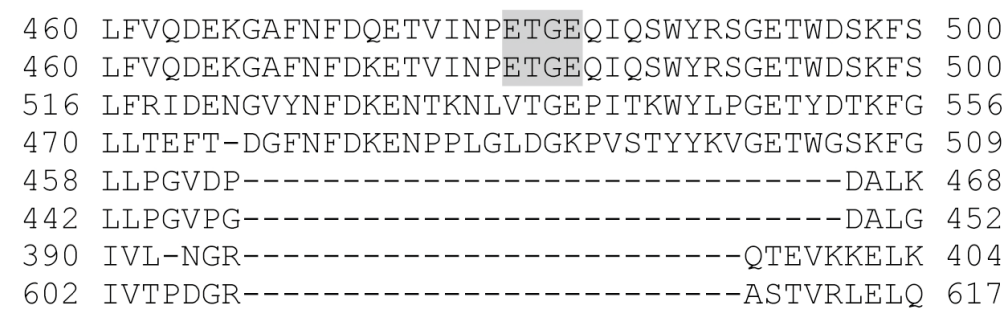

A multiple sequence alignment of the flexible loop region containing ETGE motif from different DPPs III performed by Clustal Omega 1.2.1. ${ }^{\mathrm{S} 1}$ UniProt sequence accession codes are Q9NY33 (Homo sapiens), Q99KK7 (Mus musculus), Q9VHR8 (Drosophila melanogaster), Q08225 (Saccharomyces cerevisiae), Q8A6N1 (Bacteroides thetaiotaomicron), Q7MX92 (Porphyromonas gingivalis), H1XW48 (Caldithrix abyssi) and A9TLP4 (Physcomitrella patens).

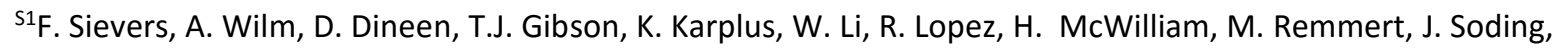
J.D. Thompson, and D.G. Higgins, Mol. Syst. Biol., 7 (2011): 539

\section{Hydrogen/deuterium exchange experiment}

For differential analysis of deuterium incorporation between unliganded forms and a complex of investigated proteins three HDX experiments were completed, with unliganded human DPP III (43 $\mu \mathrm{M}, 20 \mathrm{mM}$ tris $\mathrm{pH} 7.4$ ), unliganded kelch domain $(60 \mu \mathrm{M}, 20 \mathrm{mM}$ tris $\mathrm{pH} 7.4)$ and their complex. Stock solutions of human DPPIII protein $(57 \mu \mathrm{M}, 20 \mathrm{mM}$ tris $\mathrm{pH} 7.4)$ and kelch domain $(57 \mu \mathrm{M}, 20 \mathrm{mM}$ tris $\mathrm{pH} 7.4)$ were mixed together in equal $\mathrm{v} / \mathrm{v}$ ratio to produce $130 \mu \mathrm{L}$ of protein complex solution for HDX experiment. All experiments were carried out working on commercial HDX experimental platform. Fluidics, valves, and columns were maintained at $0.5^{\circ} \mathrm{C}$ with HDX Manager (the pepsin column was kept at $20^{\circ} \mathrm{C}$ ). $\mathrm{C} 18$ column outlet was coupled directly to the ion source of SYNAPT G2 HDMS (Waters) working in lon Mobility mode. For protein identification, mass spectra were acquired in $\mathrm{MS}^{\mathrm{E}}$ acquisition mode. Spectrometer parameters were as follows: ESI positive mode, capillary voltage $3 \mathrm{kV}$, sampling cone voltage $35 \mathrm{~V}$, extraction cone voltage $3 \mathrm{~V}$, source temperature $80^{\circ} \mathrm{C}$, desolvation temperature $175^{\circ} \mathrm{C}$, and desolvation gas flow $800 \mathrm{l} / \mathrm{h}$. Detailed experimental schematics were previously shown by Engen et. al. ${ }^{\mathrm{S2}}$. Briefly, hydrogen/deuterium exchange reaction was started by mixing $5 \mu$ l of protein stock with $45 \mu \mathrm{l}$ of D2O reaction buffer (20 mM Tris, pD 7.4), the exchange reactions were carried out for specific time points (10 s, $1 \mathrm{~min}, 20 \mathrm{~min}, 1$ and 4 hours) at room temperature. The exchange was quenched by reducing $\mathrm{pH}$ by adding the reaction mixture to stop buffer $(2 \mathrm{M}$ glycine $\mathrm{pH} 2.5)$ cooled on ice. Exchanged protein was digested on an immobilized pepsin column (Porozyme; $\mathrm{ABI}$ ), peptides were passed to the C18 trapping column (Acquity BEH C18 VanGuard pre-column, Waters) and then directed onto a reverse-phase column (Acquity UPLC BEH C18 column, Waters) with a $6-40 \%$ gradient of acetonitrile in $0.1 \%$ formic acid at $40 \mu \mathrm{l} / \mathrm{min}$ using nano Acquity binary solvent manager (Waters). All time periods were acquired in triplicate for calculating measurement error. Peptides were identified using ProteinLynx Global Server software (PLGS, Waters). Deuteration levels for each peptide resulting from the exchange were calculated with DynamX 2.0 software, (Waters). 


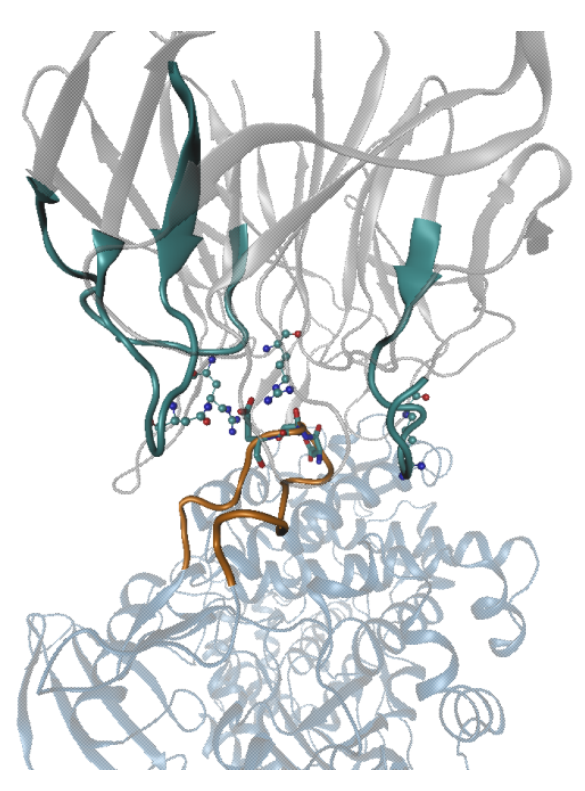

Figure. Significant difference in the HD exchange was detected in the Kelch peptides (colored green) by comparing the deuterium uptake values in unliganded protein with corresponding values obtained for the DPPIII-Kelch complex. Deuterium incorporation in the Kelch region covered by those peptides is reduced indicating stabilization of the conformation dynamic when protein is in the complex. The structure obtained after 120 ns starting from the complex GX-DPP III-closed is displayed.

DPP III and Kelch are represented as ribbons (ice-blue and gray, respectively) while the DPP III flexible loop (containing ETGE motif, shown in stick respresentation) is colored orange.

\section{A Computational Study of Human DPP III - Keap1 Interactions}

Data Analysis

$$
\begin{aligned}
& R M S D=\sqrt{\frac{\sum_{i}^{N}\left(r_{A, i}-r_{B, i}\right)^{2}}{N}} \\
& R M S F=\sqrt{\frac{1}{T} \sum_{t j=1}^{T}\left(r_{i}\left(t_{j}\right)-r_{i}^{r e f}\right)^{2}} \\
& R_{g}=\sqrt{\frac{\sum\left(R_{i}-R_{C M}\right)^{2}}{N}}
\end{aligned}
$$




\section{Tables}

TABLE S1 Systems studied by all-atom MD simulations (ff14SB force field)

\begin{tabular}{|c|c|}
\hline INITIAL COMPLEX & Simulation time (ns) \\
\hline SDA-DPP III-open & 200 \\
\hline GX'-DPP III-open & 120 \\
\hline SDA-DPP III-openSt-1 & 60 \\
\hline SDA-DPP III-openSt-2 & 57 \\
\hline GX-DPP III-closed & 120 \\
\hline GX-DPP III-closed-St & 80 \\
\hline
\end{tabular}

TABLE S2 The binding free energy components determined by MM-GBSA and MM-PBSA calculations for the DPP III (closed)-Kelch and DPP III (open)-Kelch complex.

\begin{tabular}{|c|c|c|c|c|}
\hline \multicolumn{4}{|c|}{ MM-GBSA (left) and MM_PBSA (right)energies (kJ/mol) (SD= standard deviation) } \\
\hline complex & \multicolumn{2}{|c|}{ DPP III (closed)-Kelch } & \multicolumn{2}{c|}{ DPP III (open)-Kelch } \\
\hline Energy component & Average MM-GBSA & Average MM-PBSA & Average MM-GBSA & Average MM-PBSA \\
\hline$\Delta$ G (gas) & $-7(18)$ & $-49(9)$ & $109(23)$ & $12(9)$ \\
\hline$\Delta$ G (solvent) & $-80(17)$ & $-58(8)$ & $-153(19)$ & $-83(8)$ \\
\hline$\Delta$ G (total) & $-87(5)$ & $-107(4)$ & $-44(6)$ & $-71(4)$ \\
\hline
\end{tabular}

Table S3 Human DPP III amino acid residues directly interacting with the Kelch domain in the complexes at the end of CG MD simulations starting from the closed (Gramm-X) and open (SDA) complexes. Residues in blue are found in closed (Gramm-X) and open (SDA) complexes, while residues in red are found in closed (Gramm-X) and closed (SDA-variant) complexes. Residues given in bold interact with Kelch in all three complexes.

\begin{tabular}{|l|l|l|l|}
\hline Gramm-X (closed) & SDA (open) & Gramm-X (closed) & SDA ((closed) \\
\hline GLY 467 & GLU 356 & THR 481 & PRO 479 \\
\hline ALA 468 & PRO 360 & ARG 624 & GLU 480 \\
\hline GLN 473 & THR 362 & VAL 627 & THR 481 \\
\hline GLU 474 & LYS 365 & LEU 628 & GLY 482 \\
\hline THR 475 & PHE 368 & & GLU 483 \\
\hline VAL 476 & GLN 463 & ASP 633 & GLN 484 \\
\hline ILE 477 & GLU 465 & VAL 634 & GLN 486 \\
\hline ASN 478 & LYS 466 & ALA 635 & TRP 488 \\
\hline PRO 479 & PHE 469 & GLY 636 & LYS 498 \\
\hline GLU 480 & PHE 471 & & ARG 577 \\
\hline GLY 482 & ASP 472 & & GLU 581 \\
\hline GLU 483 & GLU 474 & & \\
\hline GLN 484 & THR 475 & & ARG 624 \\
\hline ILE 485 & GLU 480 & & VAL 627 \\
\hline SER 487 & GLY 482 & & LEU 628 \\
\hline TRP 488 & GLU 483 & & \\
\hline ARG 490 & GLN 484 & & ASP 633 \\
\hline ARG 620 & ILE 485 & & VAL 634 636 \\
\hline ARG 623 & GLN 486 & & \\
\hline THR 631 & TRP 488 & & \\
\hline
\end{tabular}




\section{Figures}
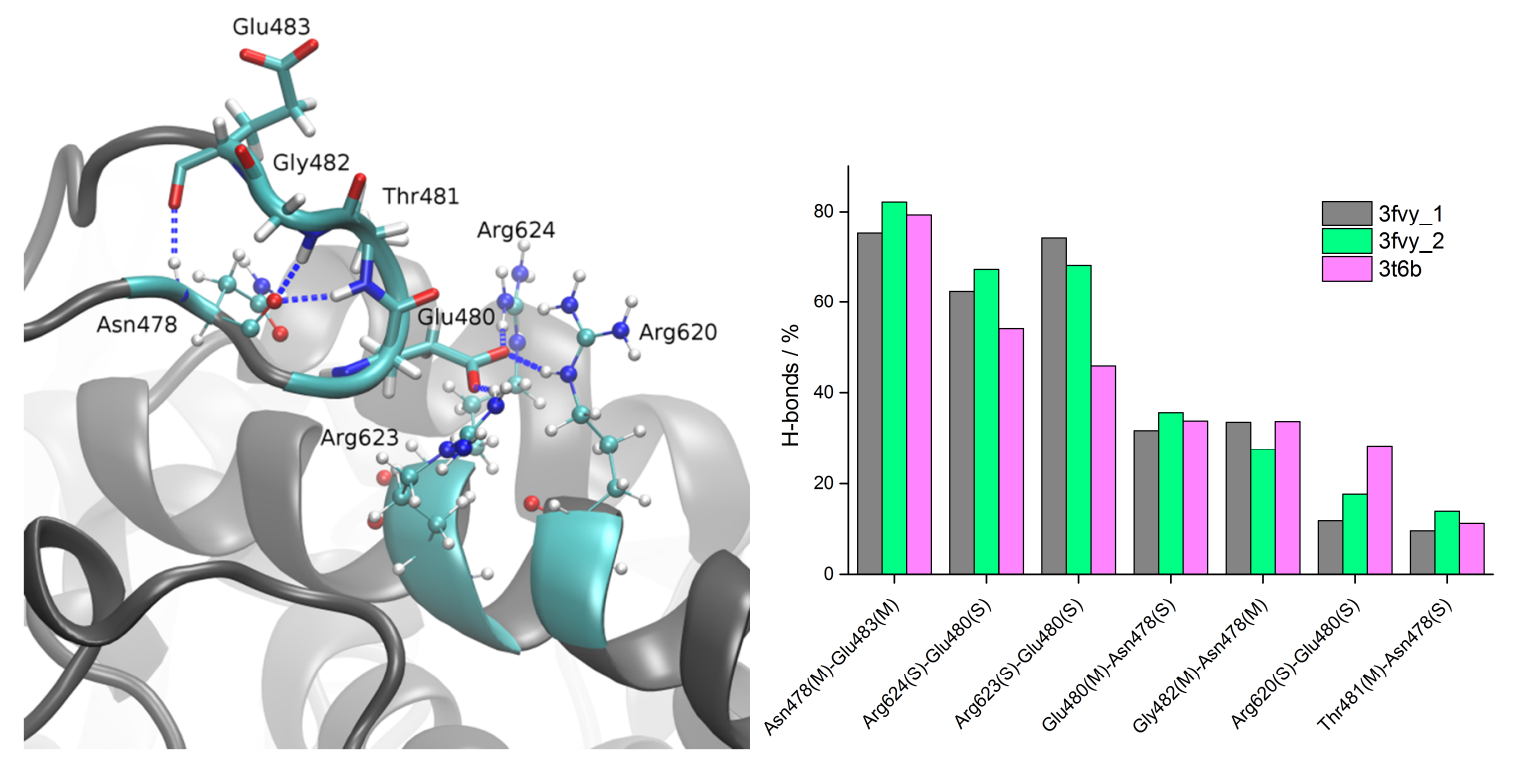

Figure S1. Left: snapshot taken from the 100-ns-long MD simulations of closed human DPP III showing hydrogen bonds that "ETGE" motif (show as sticks) makes with adjacent amino acids (shown as ball and sticks) belonging to $\alpha$-helix from the upper protein domain (helix $\alpha 23$ according to A. Tomić et al. J. Chem. Inf. Model. 2012 , 52, 1583). Right: percentage of the hydrogen bonds formed between selected amino acid pairs (amino acid pairs that make $\mathrm{H}$-bond more than $10 \%$ of simulation time) during MD simulations, with enzyme initially in its open (3fvy_1 and 3fvy_2) and closed (3t6b) form. Letters $S$ and $M$ in parentheses indicate whether the amino acid side chain or main chain participates in hydrogen bond formation, respectively.

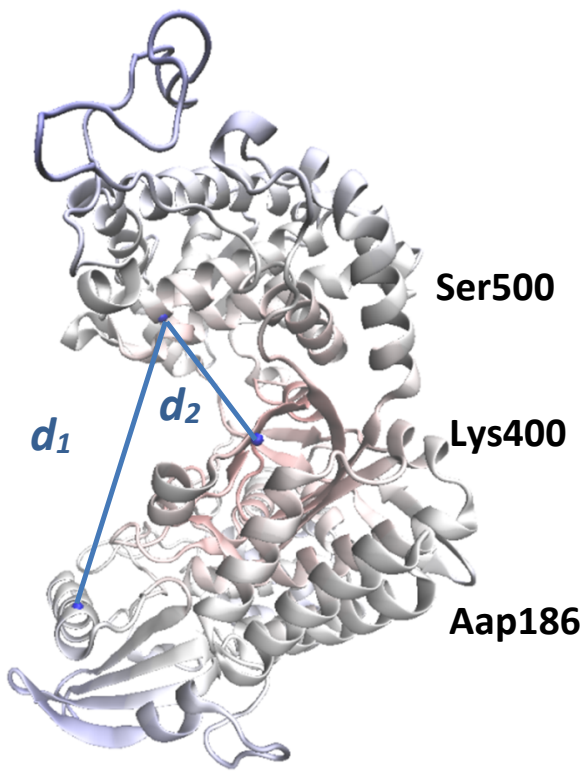

\begin{tabular}{|c|c|c|}
\hline geometric parameters & oWT & cWT \\
\hline$R_{\mathrm{g}} / \AA$ & 27.2 & 24.7 \\
$d_{1}(\mathrm{D} 186-\mathrm{S} 500) / \AA$ & 38.5 & 11.6 \\
$d_{2}(\mathrm{Q} 400-\mathrm{S} 500) / \AA$ & 25.2 & 20.9 \\
\hline
\end{tabular}

Figure S2. Distances $d_{1}$ and $d_{2}$ between the $C_{\alpha}$ atoms of Asp186 and Ser500, and Lys400 and Ser500, respectively displayed on the 3D structure of DPP III in its open form (oWT). Values of geometrical parameters, radius of gyration, and $\mathrm{d} 1$ and $\mathrm{d} 2$ distances in the experimentally determined structures (open, oWT and closed, cWT) are given in the table on the right. 

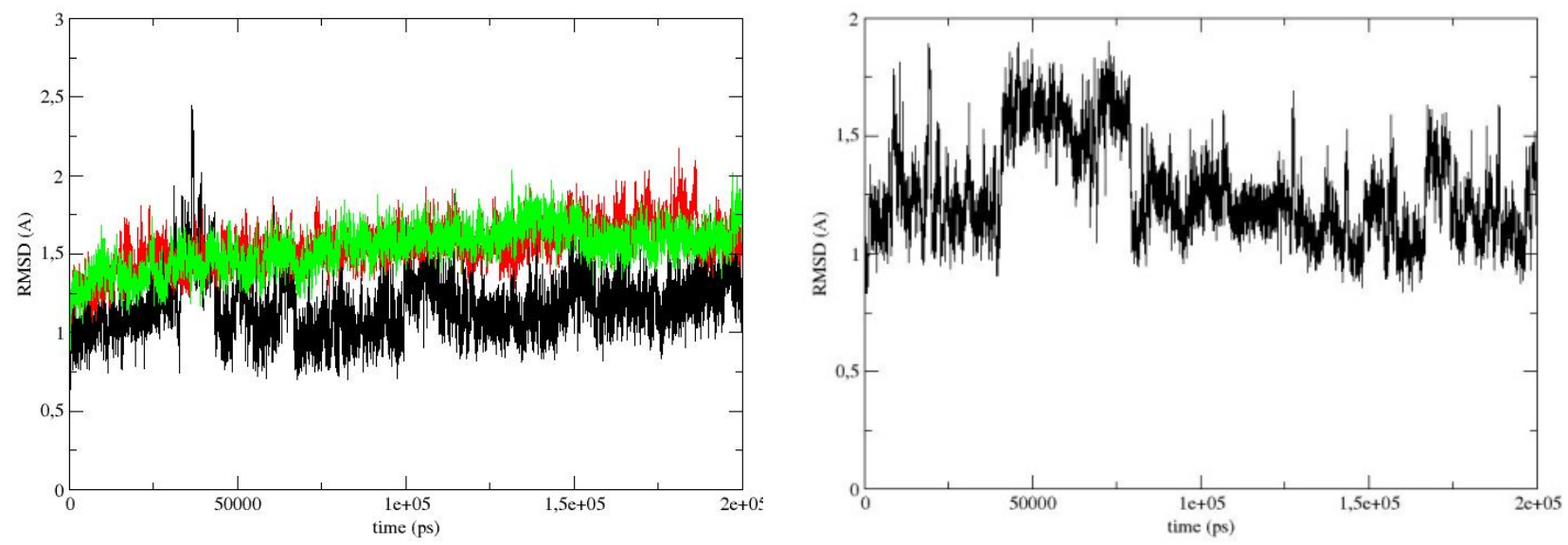

Figure S3. RMSD values determined during $200 \mathrm{~ns}$ of MD simulations of the DPP III - Kelch complex, with initial DPP III in its open form. Left RMSD of h.DPP III domains: lower (red) and upper (without flexible loop, green) and the flexible loop (black). Right RMSD of the Keap1 Kelch domain.
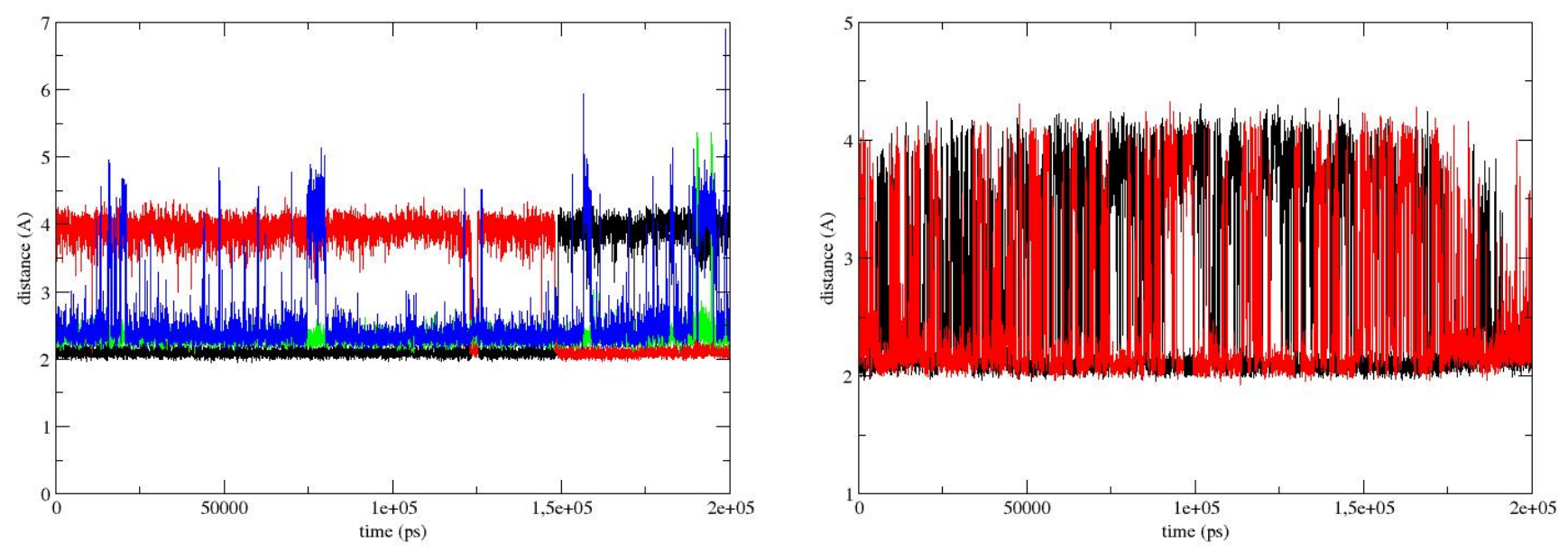

Figure S4. Coordination of the zinc ion during MD simulations with DPP III in its open form (initial complex obtained by the SDA docking server). LEFT: coordination by E451, OE1 and OE2, is represented by black and red lines, respectively. Coordination by $\mathrm{H} 450$ and $\mathrm{H} 455$ is represented by green and blue lines, respectively. RIGHT: $\mathrm{Zn}^{2+}$ coordination by E508 (OE1 and OE2, black and red lines, respectively). 


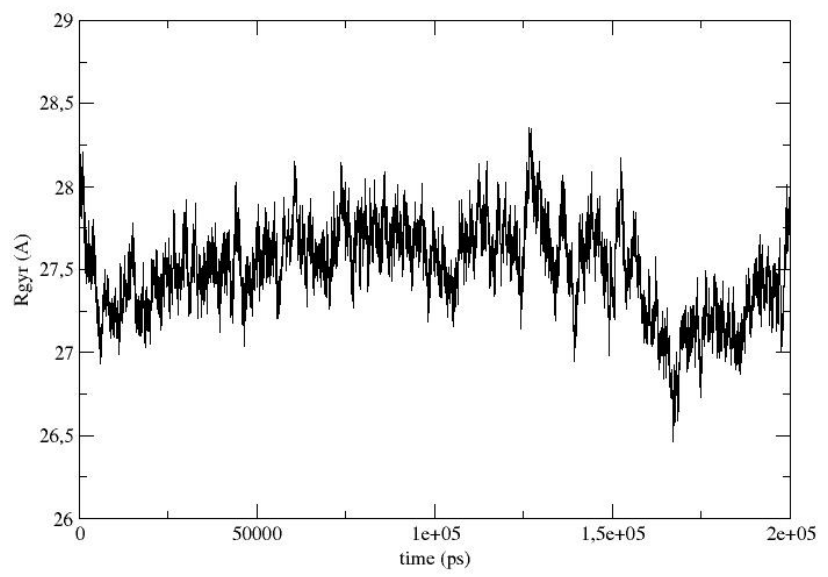

Figure S5. Radius of gyration of DPP III traced during 200 ns of MD simulations of the DPP III - Kelch complex, with initial DPP III in its open form.

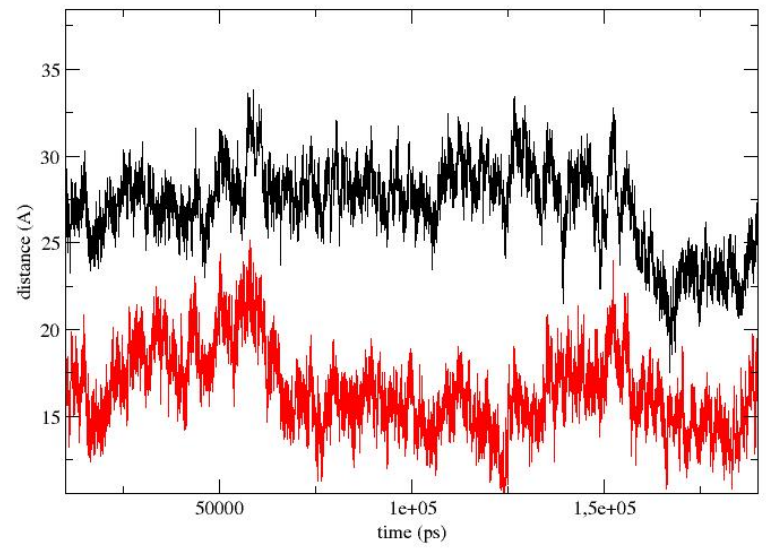

Figure S6. Distances d1 and d2 traced during 200 ns of MD simulations of the DPP III - Kelch complex, with initial DPP III in its open form. 

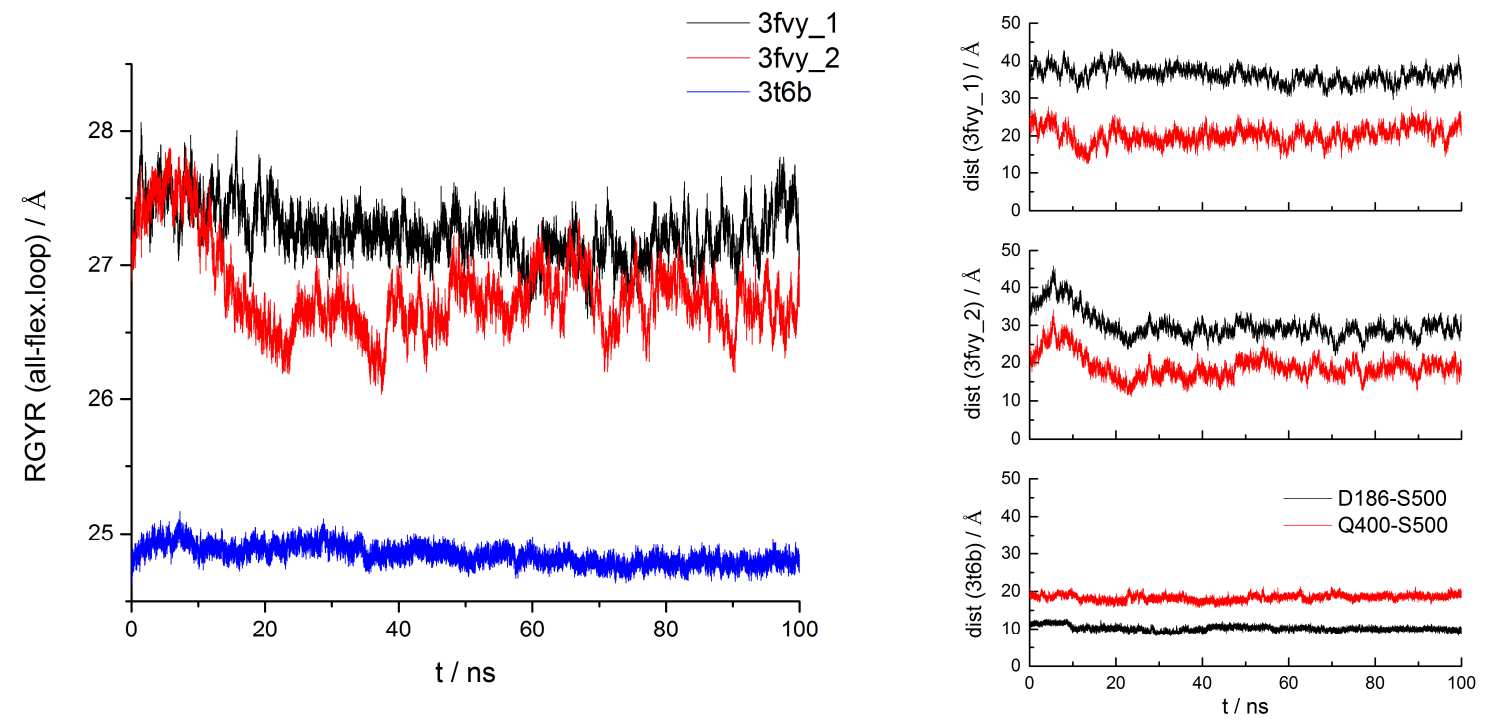

Figure S7. Left: Radius of gyration profiles obtained from MD simulations of the open (3fvy_1 and 3fvy_2) and closed (3t6b) human DPP III structures. All residues except the one from the protein flexible loop (residues 459492) were considered. Right: change in distances between D186-S500 (d1, black line) and Q400-S500 (d2, red line) $C \alpha$ atoms during the simulations.

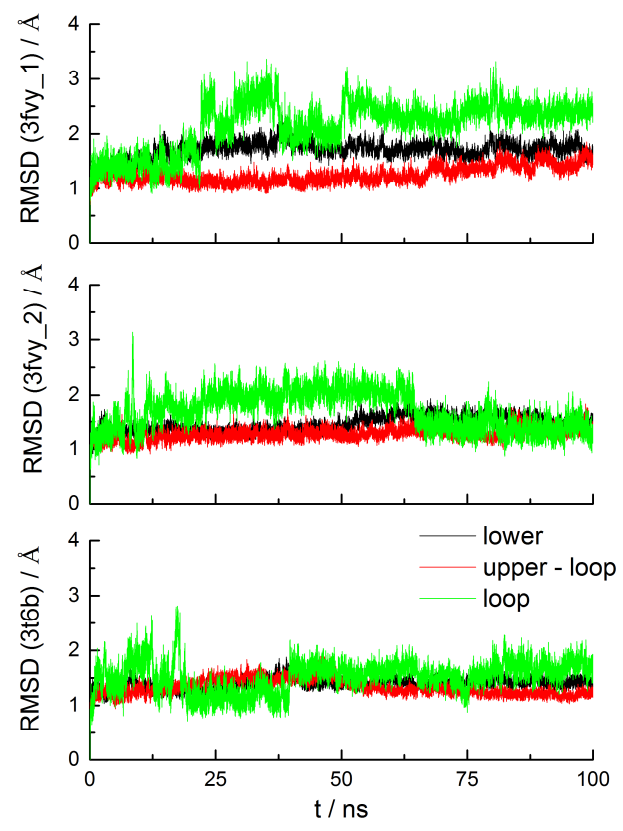

Figure S8. RMSD values determined during three $100 \mathrm{~ns}$ long MD simulations of the human DPP III (utilizing ff12sb force field), with enzyme initially in its open (3fvy_1 and 3fvy_2) and closed (3t6b) form. Calculations were performed separately for different protein parts: lower domain (black), upper domain (without flexible loop, red) and the flexible loop (green, residues 459-492). 


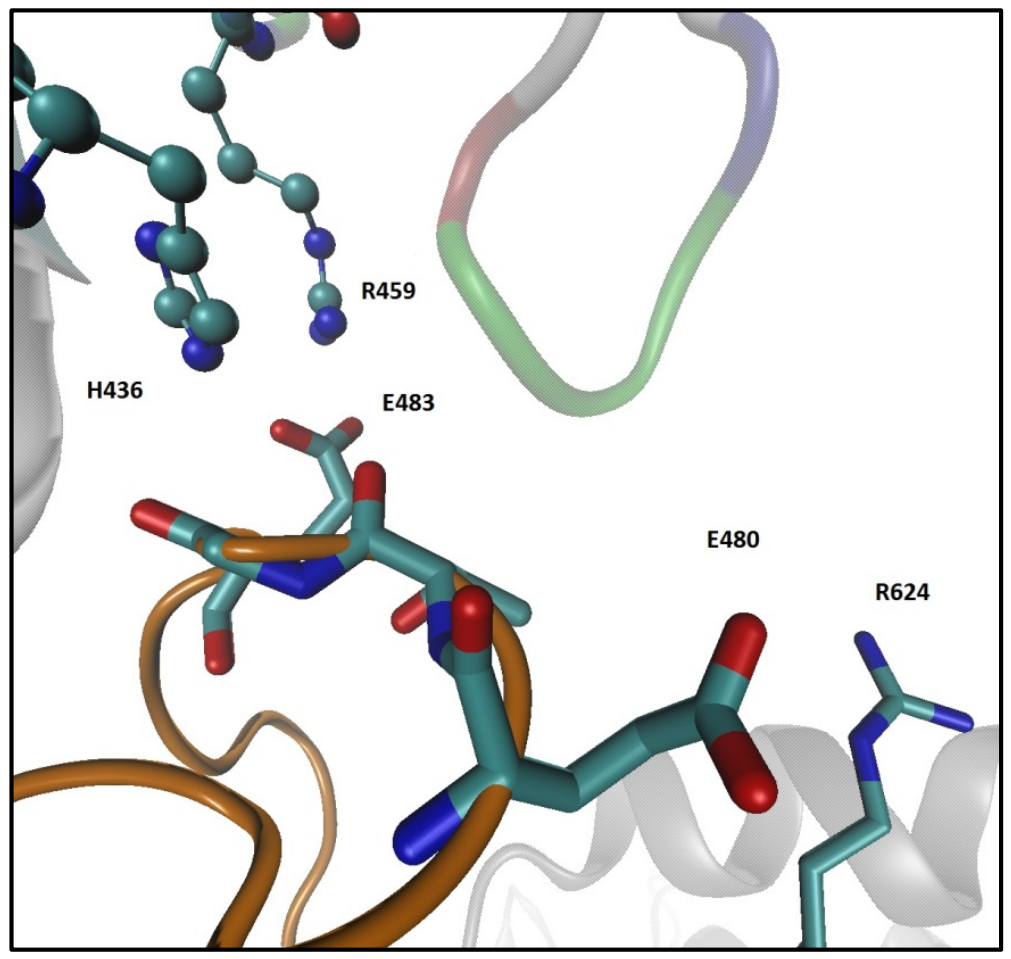

Figure 59. h.DPP III - Kelch interactions at the end of 60 ns of unrestrained MD simulations with a starting structure obtained by restrained simulations (Fig. 7). Amino acid residues from the ETGE motif are shown in stick, and the residues from the Kelch domain with which they interact with are shown as ball and stick.

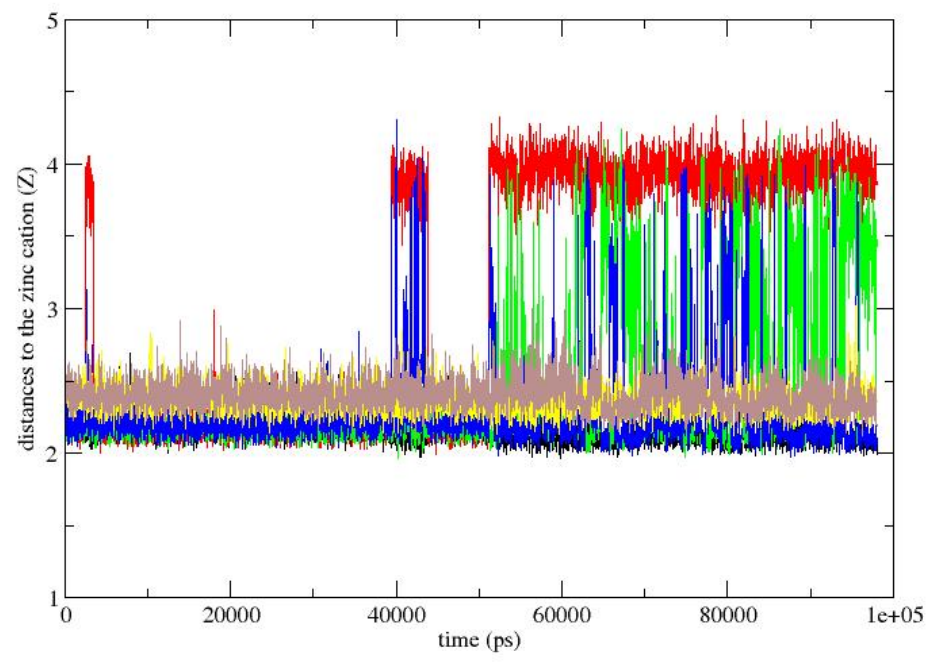

Figure S10. Coordination of the zinc ion during 120 ns of MD simulations with DPP III in its closed form (initial complex GX-DPP III-closed). Coordination by $\mathrm{H} 450$ and $\mathrm{H} 455$ is represented by blue and yellow lines, respectively; coordination by $\mathrm{E} 451, \mathrm{OE} 1$ and $\mathrm{OE2}$, is represented by black and red lines, respectively; coordination by E508 (OE1 and OE2, brow and green lines, respectively). 

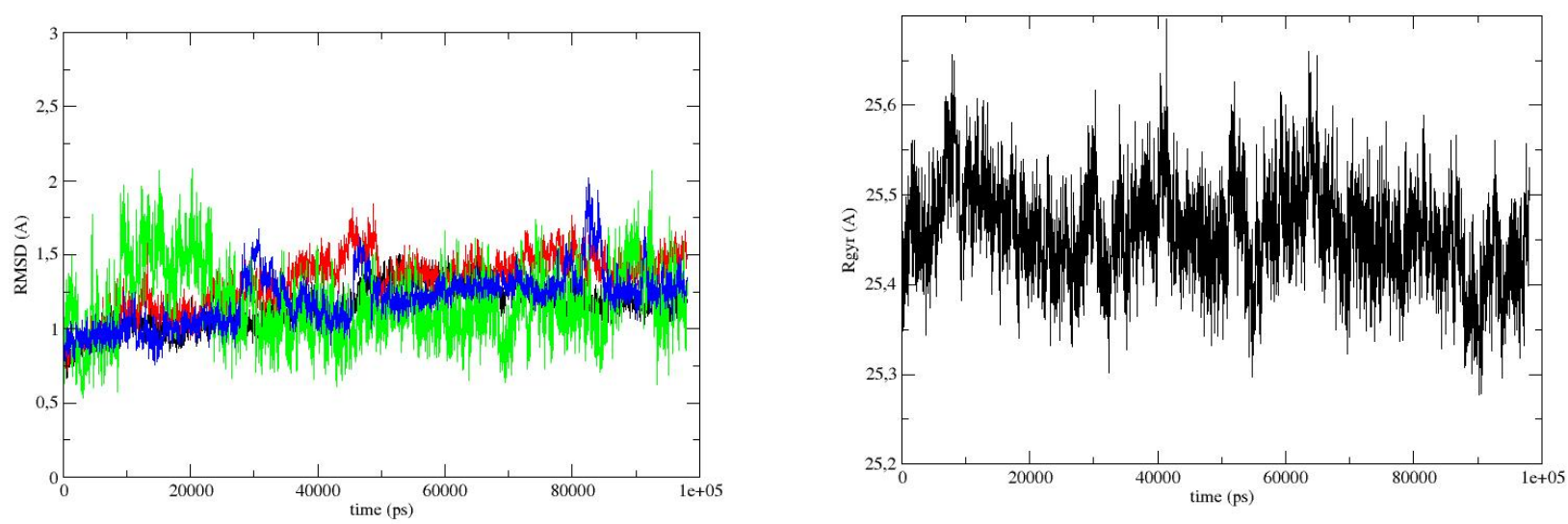

Figure S11. RMSD (left) and Radius of gyration (right) values determined during 120 ns of MD simulations of the DPP III - Kelch complex, with initial DPP III in its closed form. Left: RMSD (Å) of DPP III domains: lower (red) and upper (without flexible loop, black) and the flexible loop (blue and of the Keap1 Kelch domain (green)); left: $\operatorname{Rgyr}(\AA ̊)$ of DPP III. 

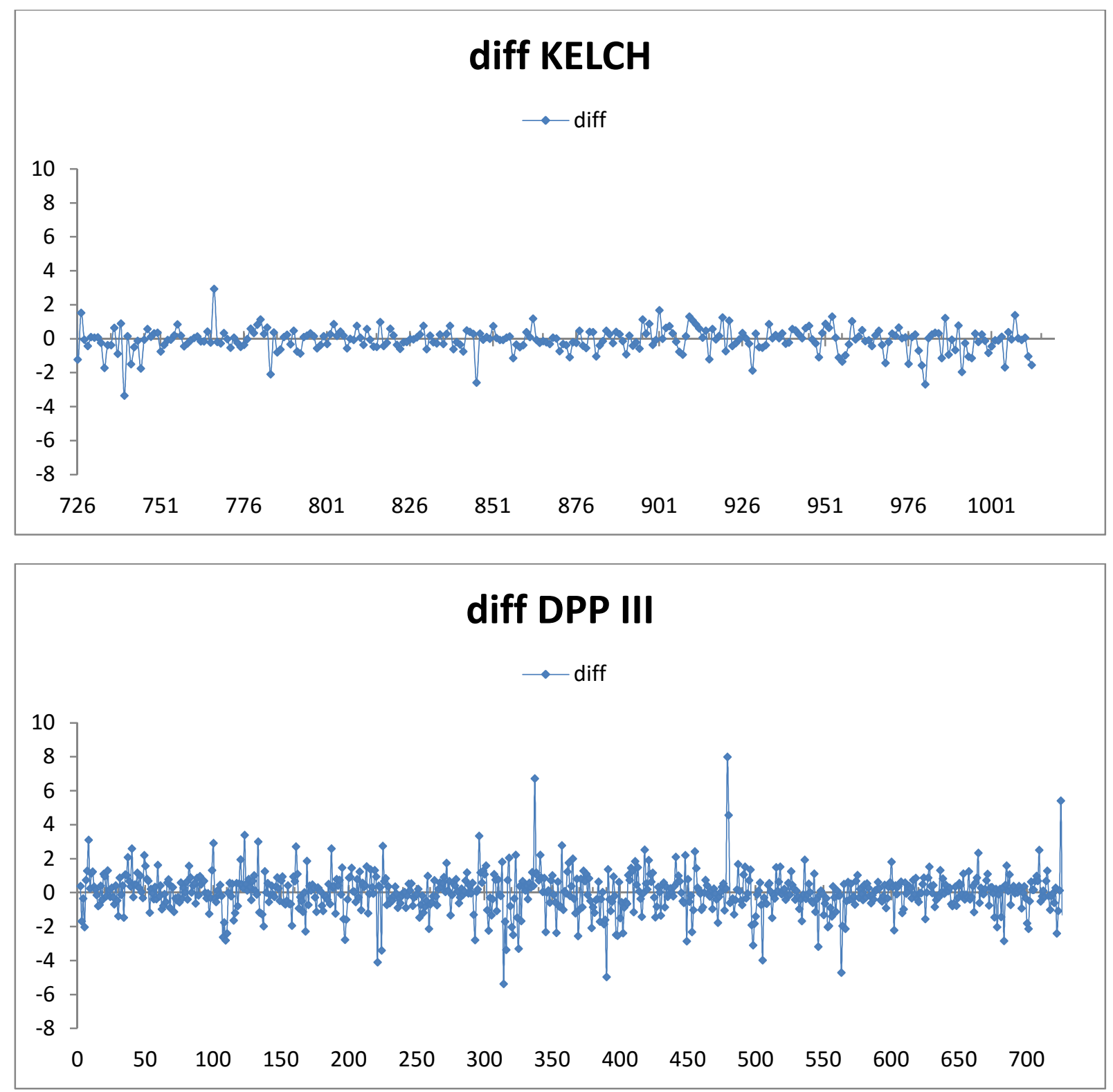

Figure S12. Differences of the residue (i) based free energies determined for the DPP III-Kelch complexes with DPP III in its closed and open form, $\Delta \Delta \mathrm{Gi}(\mathrm{kJ} / \mathrm{mol})=\Delta \Delta \mathrm{Gi}^{\text {closed }}(\mathrm{kJ} / \mathrm{mol})-\Delta \Delta \mathrm{Gi}^{\text {open }}(\mathrm{kJ} / \mathrm{mol})$ 


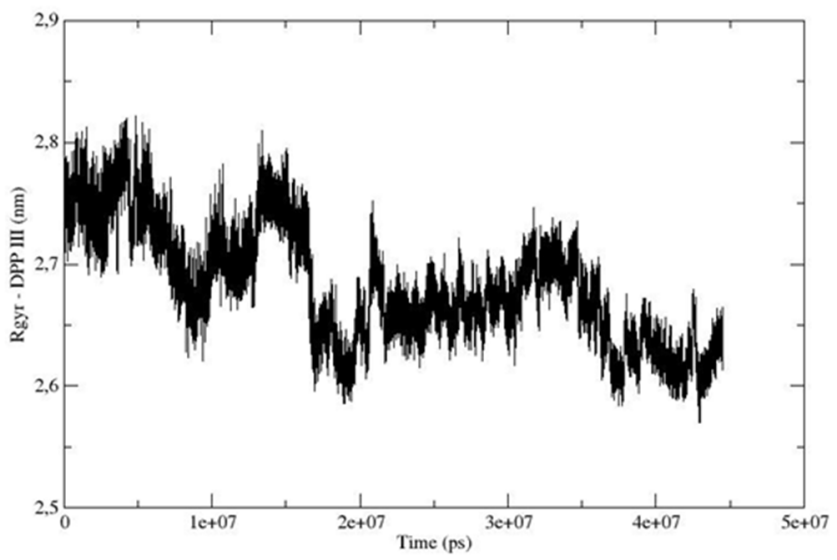

Figure S13. Radius of gyration of DPP III traced during $44 \mu$ s of CG simulation of the DPP III - Kelch complex, with initial DPP III in its open form.

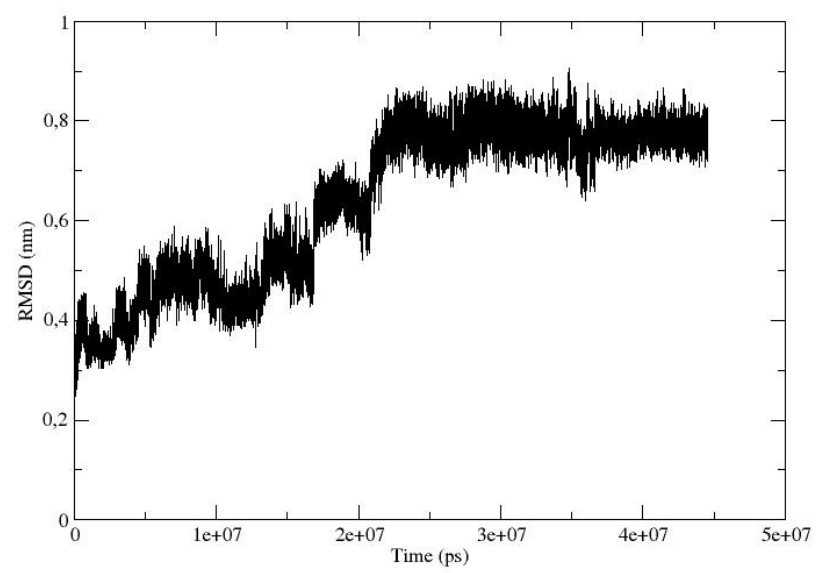

Figure S14. RMSD of h.DPP III traced during $44 \mu$ s of CG simulation of the DPP III - Kelch complex, with initial DPP III in its open form. 

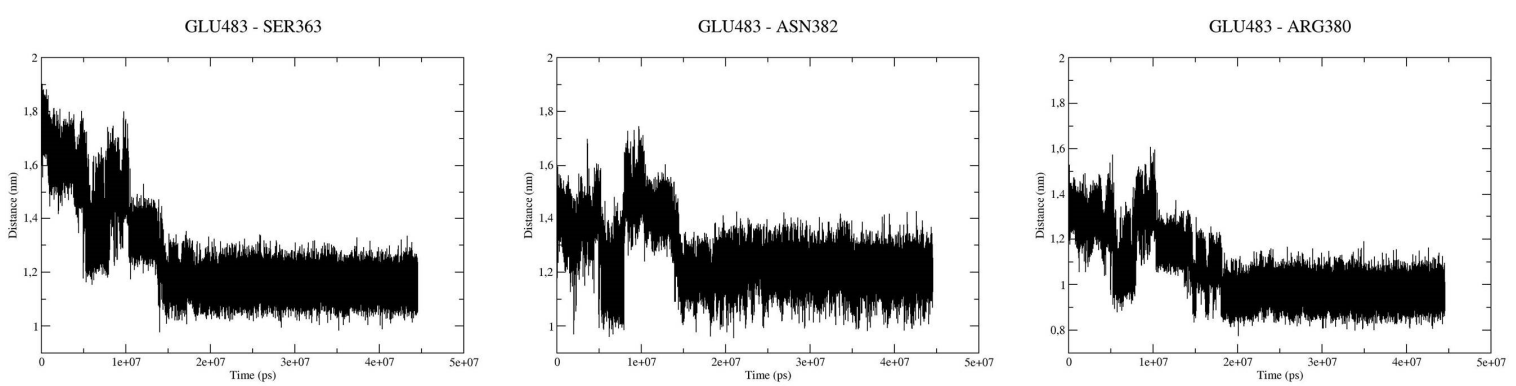

GLU480 - SER508
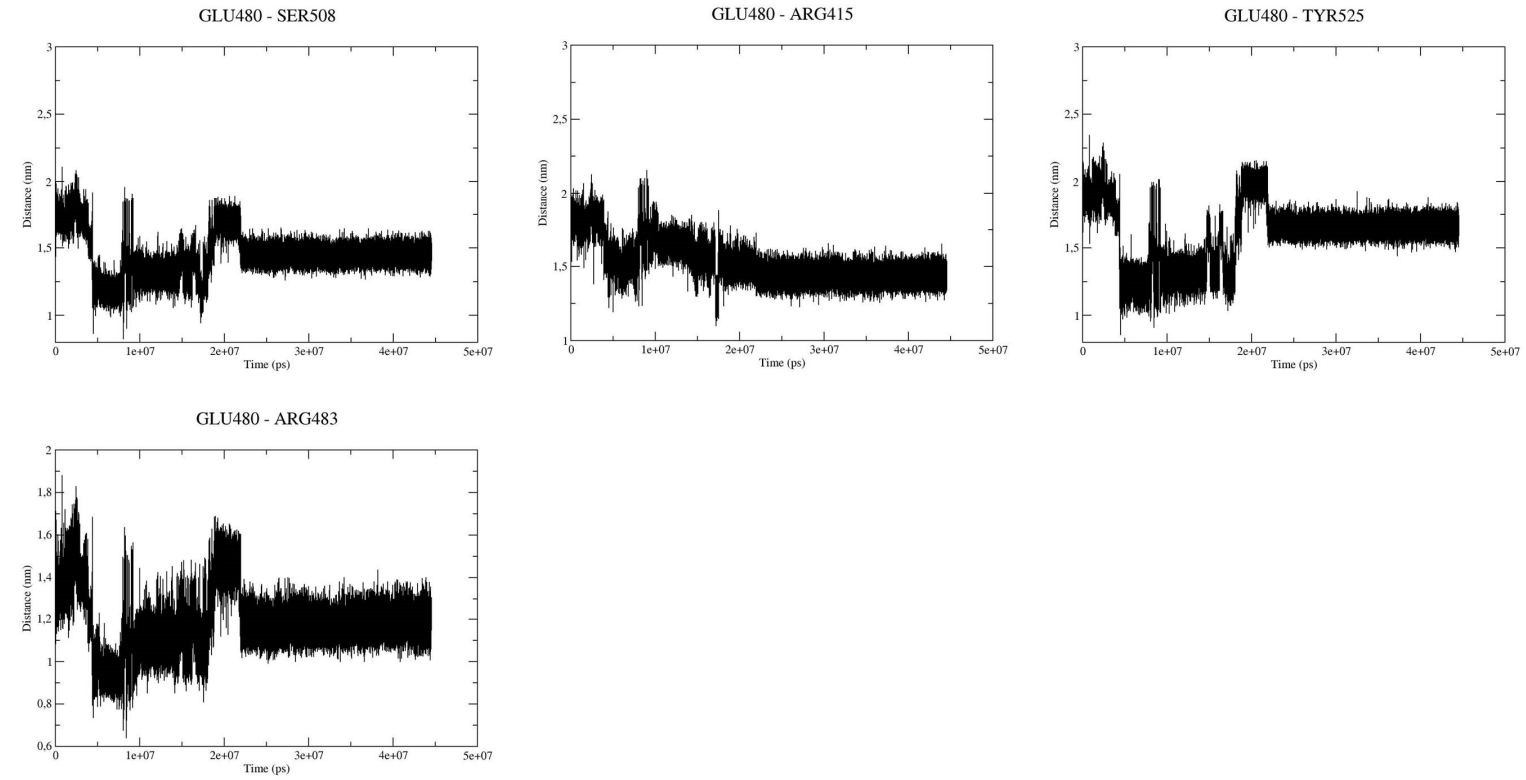

Figure S15. Distances of interacting residues predicted as stabilizing factors by Hast et al. between DPP III (residues GLU483 and GLU480) and Keap1 Kelch (residues SER363, ASN382, ARG380, SER508, ARG415, TYR525, ARG483) for simulation time of $44 \mu \mathrm{s}$, with initial DPP III in its open form.

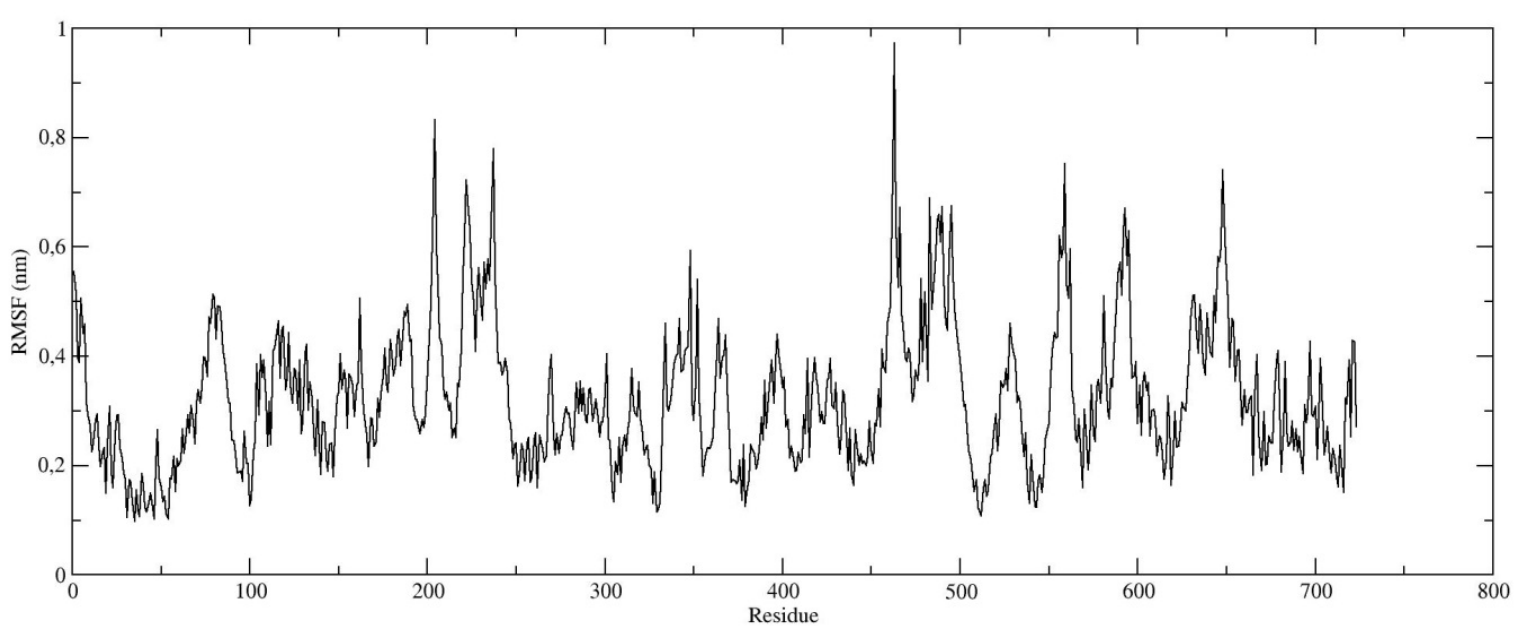

Figure S16. RMSF per residue for DPP III traced during $44 \mu$ s of CG simulation of the DPP III - Kelch complex, with initial DPP III in its open form. 


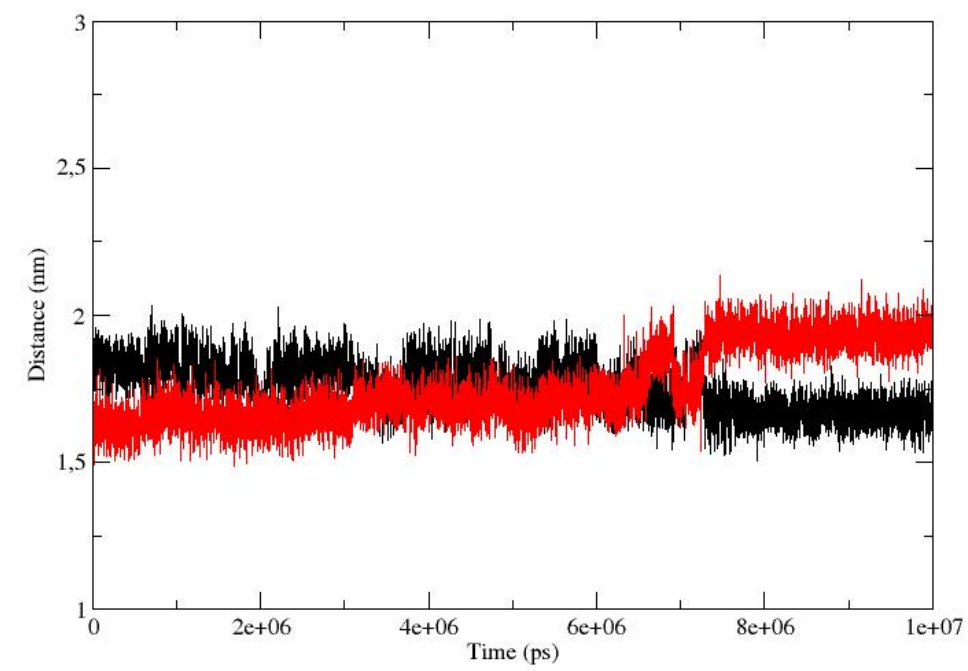

Figure S17. Distances d1 (black) and d2 (red) traced during $10 \mu$ s of CG simulation of the DPP III - Kelch complex (GRAMM-X variant), with initial DPP III in its closed form.

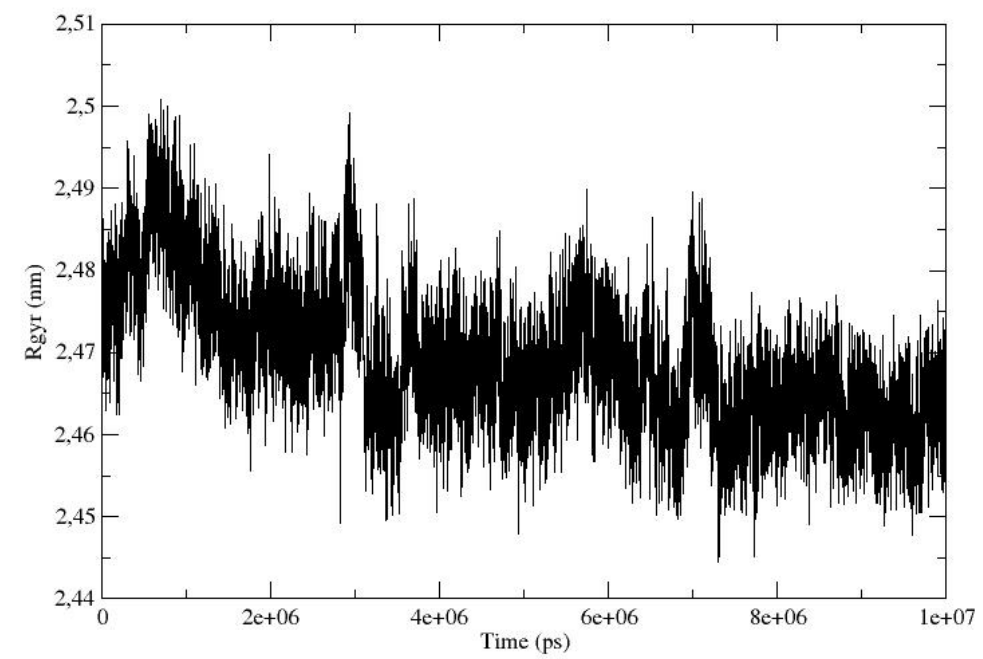

Figure S18 Radius of gyration of DPP III traced during $10 \mu$ s of CG simulation of the DPP III - Kelch complex (GRAMM-X variant), with initial DPP III in its closed form. 
GLU483 - SER363

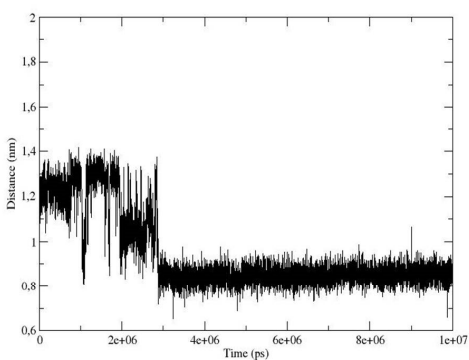

GLU480 - SER508

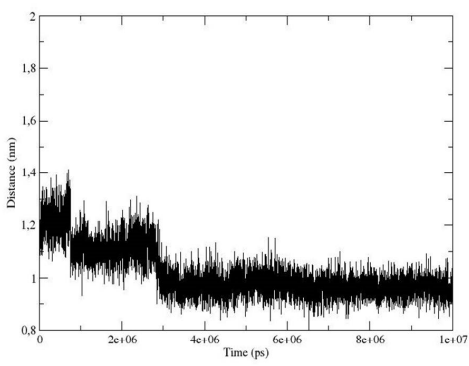

GLU480 - ARG483

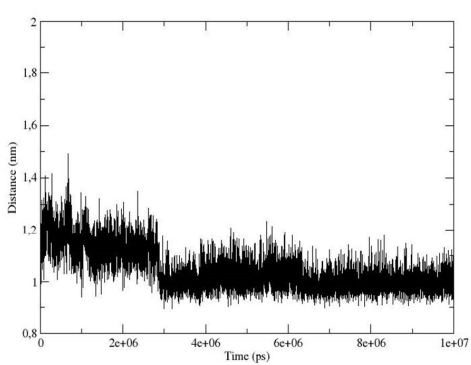

GLU483 - ASN382

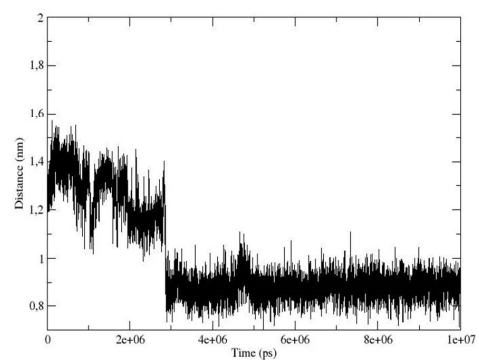

GLU480 - ARG415

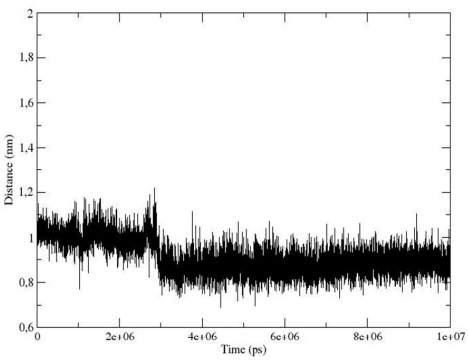

GLU483 - ARG380

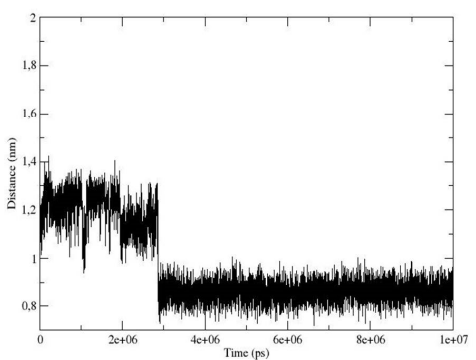

GLU480 - TYR525

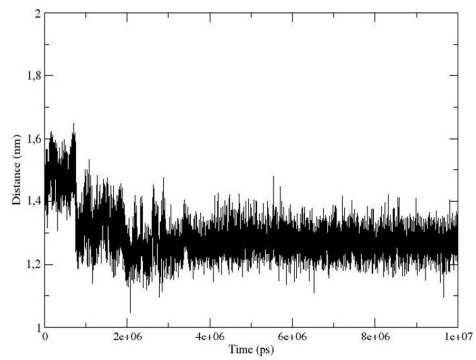

Figure S19. Distances of interacting residues predicted as stabilizing factors by Hast et al. between DPP III (residues GLU483 and GLU480) and Keap1 Kelch (residues SER363, ASN382, ARG380, SER508, ARG415, TYR525, ARG483) for simulation time of $10 \mu \mathrm{s}$, with initial DPP III in its closed form (GRAMM-X variant). 


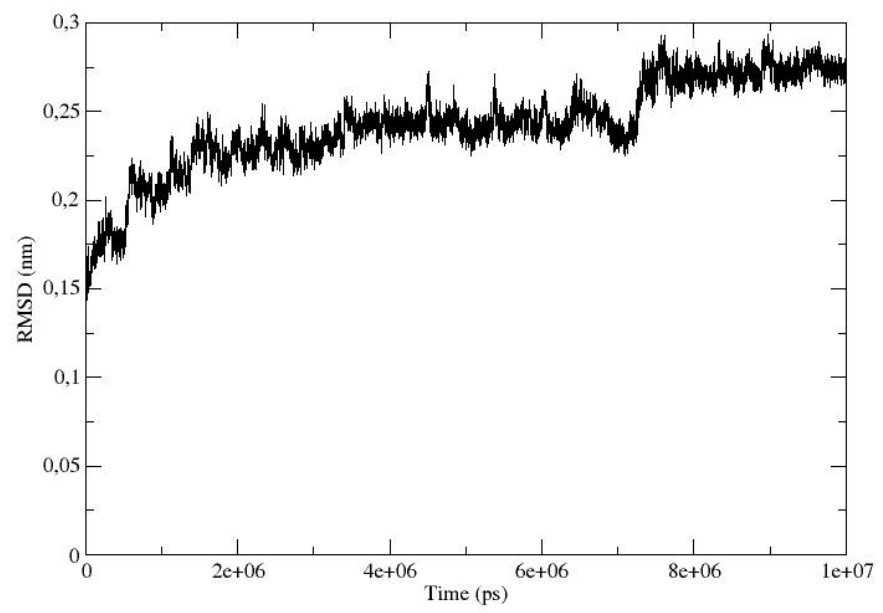

Figure S20. RMSD of h.DPP III traced during $10 \mu \mathrm{s}$ of CG simulation of the DPP III - Kelch complex (GRAMM-X variant), with initial DPP III in its closed form.

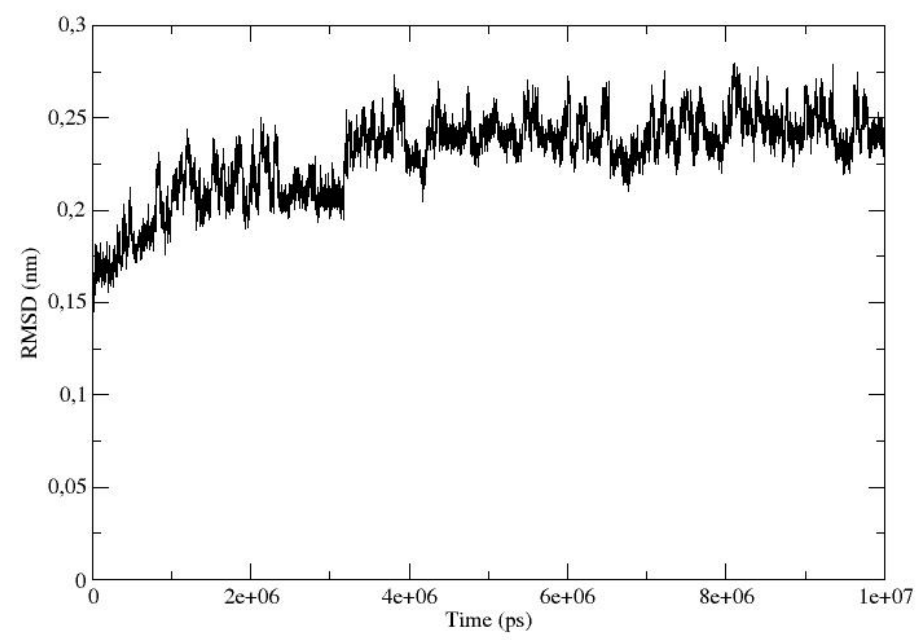

Figure S21. RMSD of h.DPP III traced during $10 \mu$ s of CG simulation of the DPP III - Kelch complex (SDA variant), with initial DPP III in its closed form. 

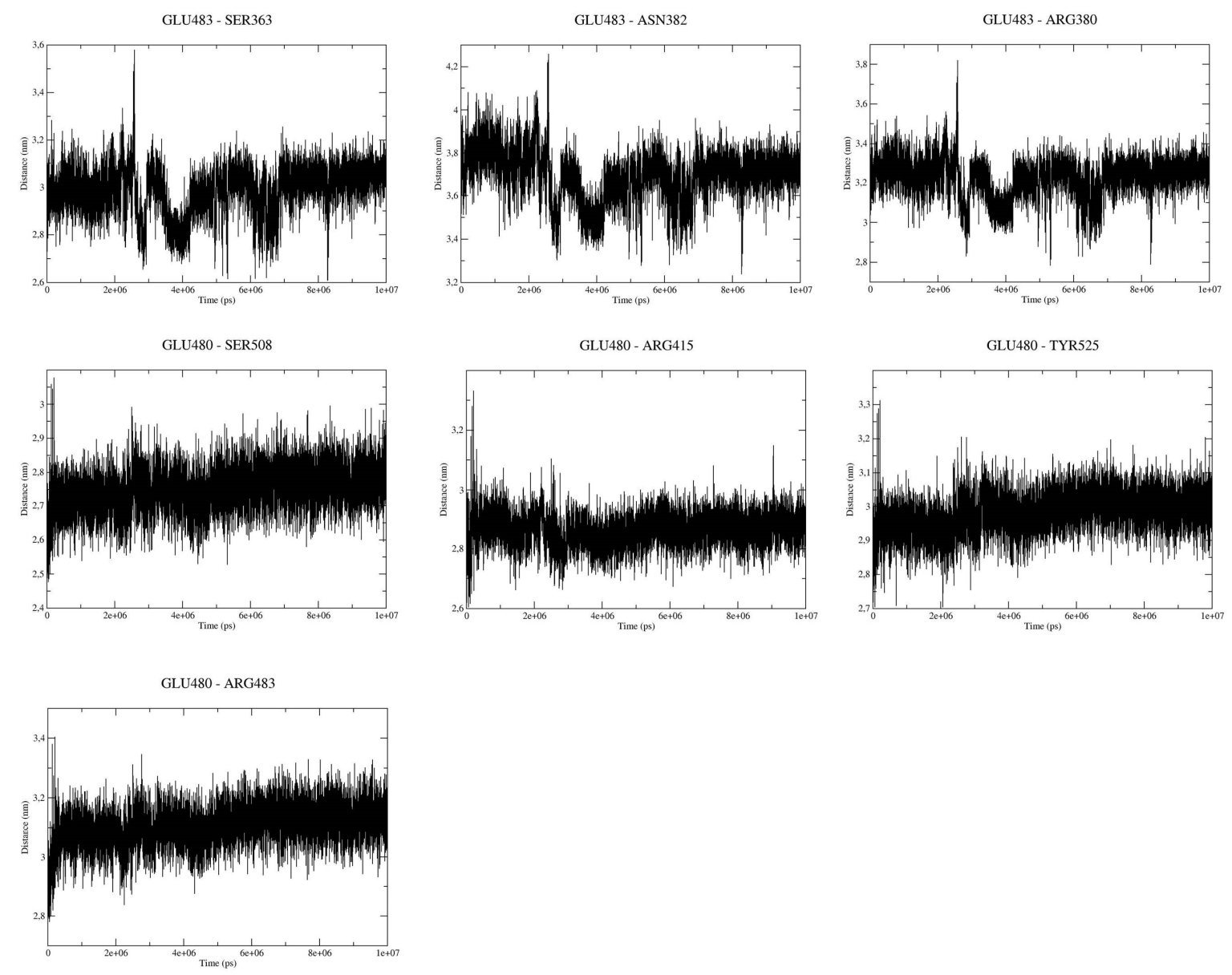

Figure S22. Distances of interacting residues predicted as stabilizing factors by Hast et al. between DPP III (residues GLU483 and GLU480) and Keap1 Kelch (residues SER363, ASN382, ARG380, SER508, ARG415, TYR525, ARG483) for simulation time of $10 \mu \mathrm{s}$, Initial DPP III in its closed form, web-SDA variant.

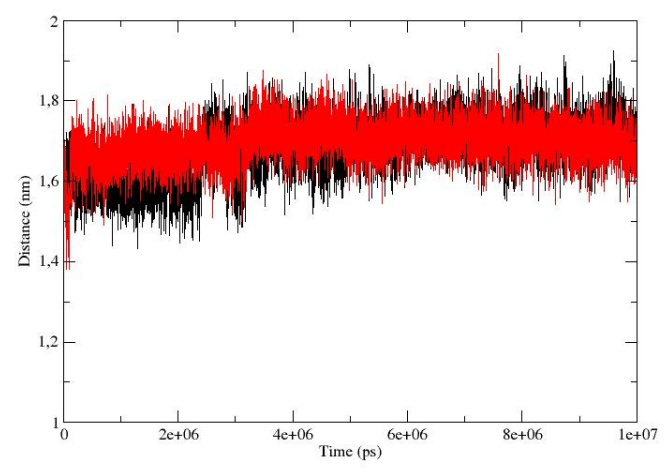

Figure S23. Distances $d_{1}$ (black) and $d_{2}$ (red) for DPP III in „closed“ complex (SDA variant) for simulation time of $10 \mu \mathrm{s}$. 


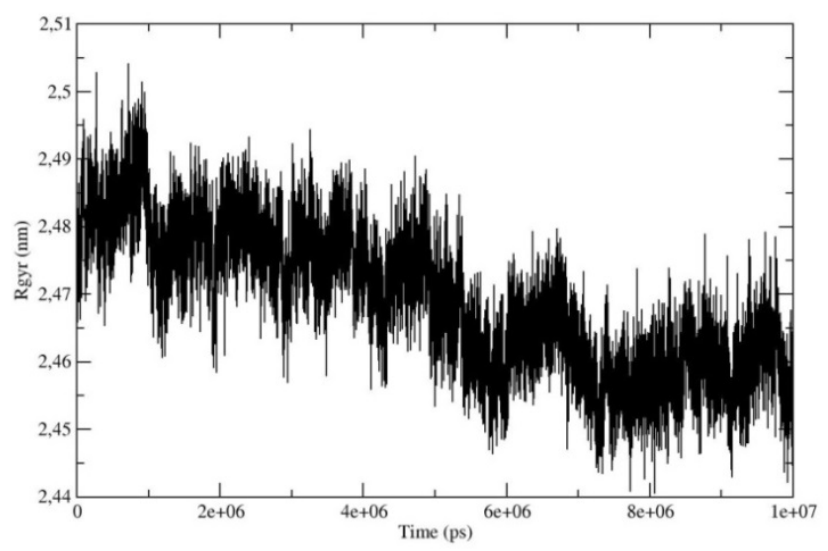

Figure S24. Radius of gyration of DPP III traced during $10 \mu$ s of CG simulation of the DPP III - Kelch complex (SDA variant), with initial DPP III in its closed form.

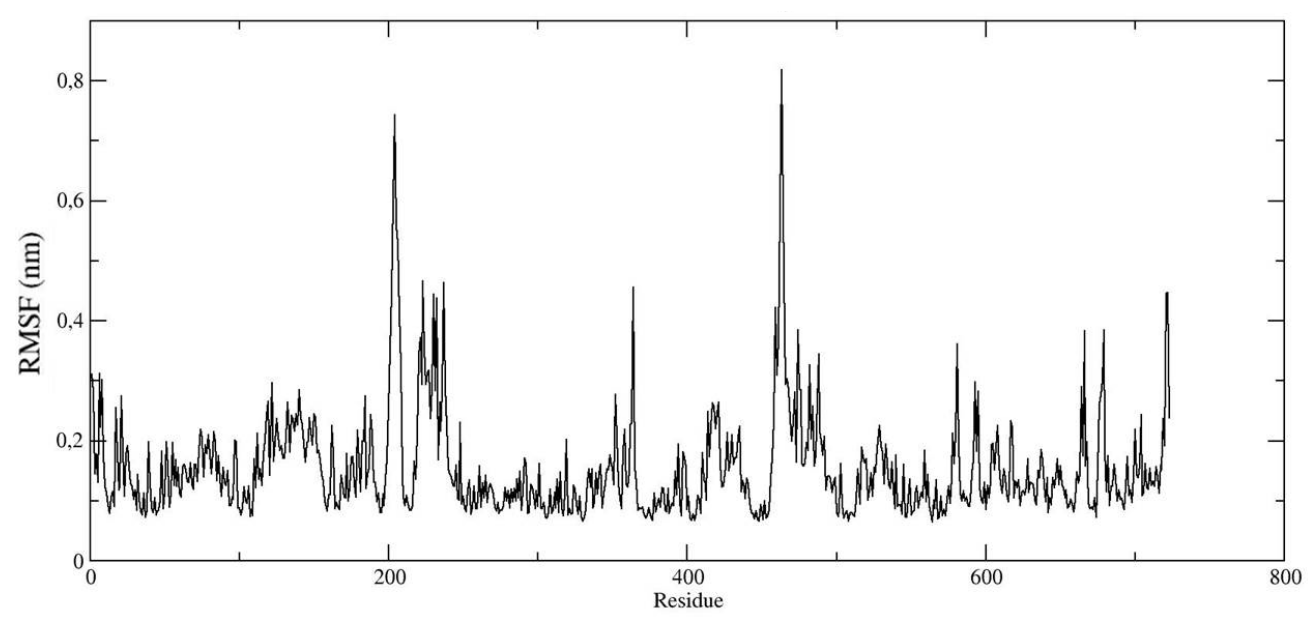

Figure S25. RMSF per residue for DPP III traced during $10 \mu$ s of CG simulation of the DPP III - Kelch complex (SDA variant), with initial DPP III in its closed form. 


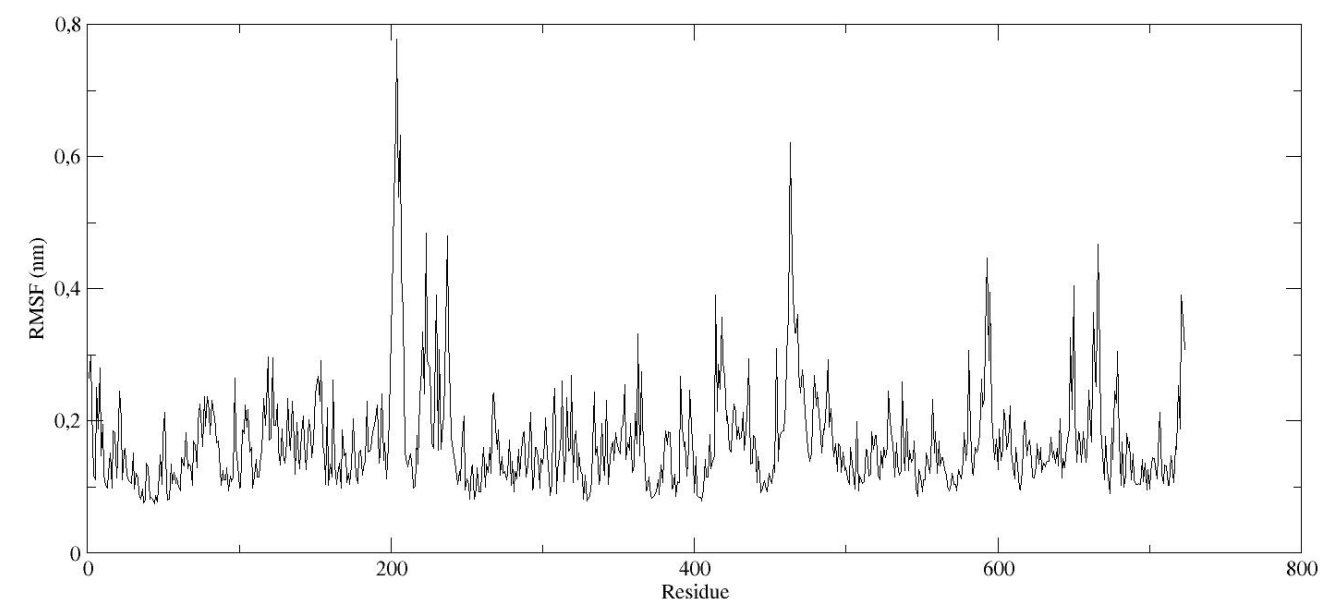

Figure S26. RMSF per residue for DPP III traced during $10 \mu$ s of CG simulation of the DPP III - Kelch complex (GRAMM-X variant), with initial DPP III in its closed form. 


\section{All atom MD simulations with the initial structure predicted by GRAMM-X server (Figure 3, middle).}

Simulations with DPP III bound to the more structured, wider, side of the southpaw shaped six-blade $\beta$ propeller Kelch domain resulted also with the stable structure (see Figure below), as well as the conserved coordination of the zinc ion. However, such a complex is not in line with the results of HD exchange experiment, so details of the simulations have not been presented in this paper.

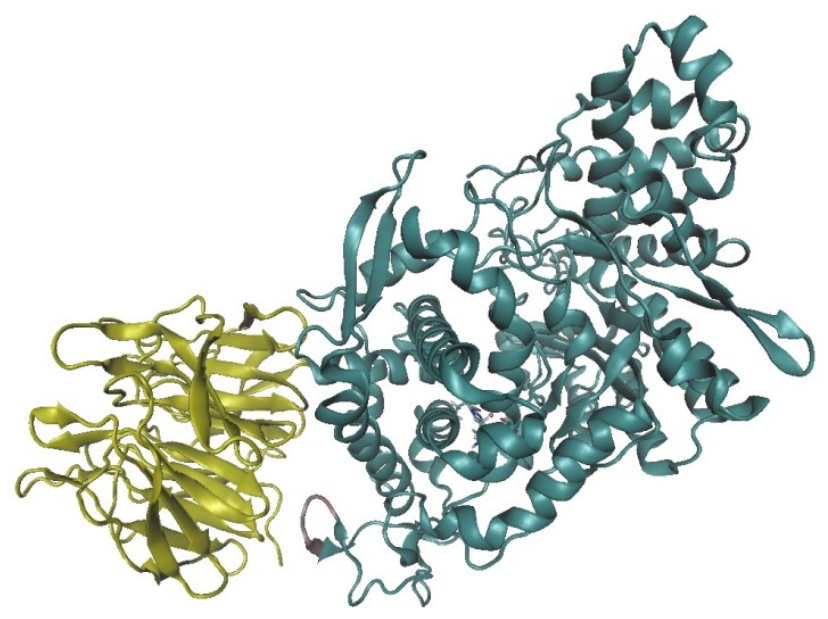

Final structure of complex with DPP III bound to the more structured, wider, side of the southpaw shaped sixblade $\beta$-propeller Kelch domain obtained after 80 ns of MD simulations at room temperature. The ETGE motif is colored pink.

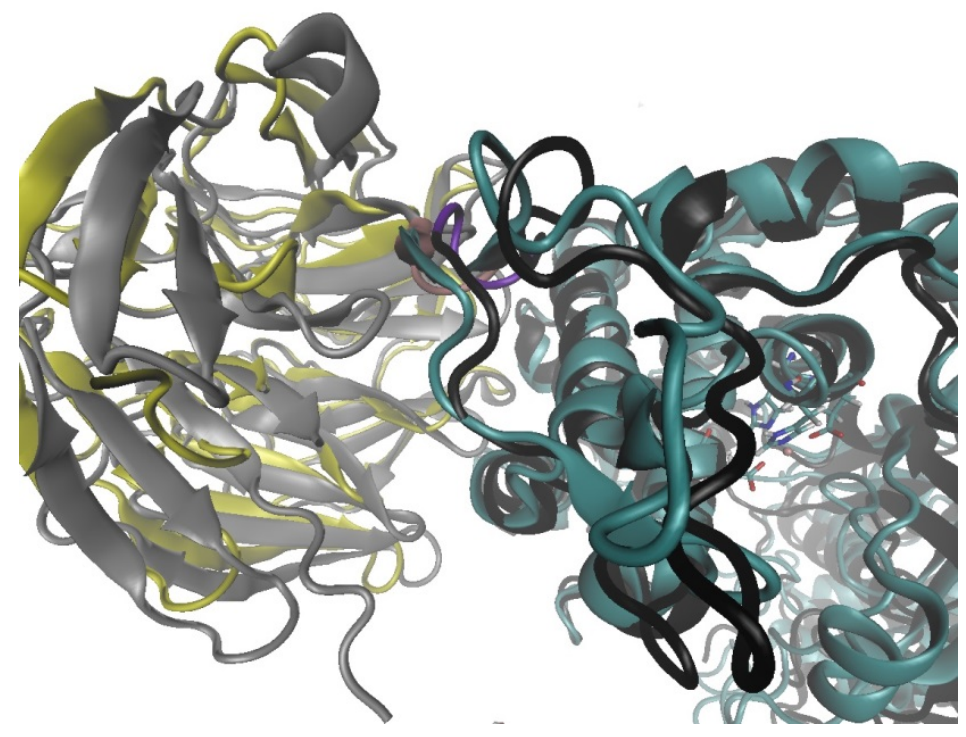

Aligned structures obtained after the equilibration (Kelch and DPP III colored black and gray, respectively) and after $80 \mathrm{~ns}$ of MD simulations (Kelch and DPP III colored yellow and cyan, respectively). The $\mathrm{Zn}^{2+}$ (pink sphere) coordination in the final structure is displayed (the zinc ligand are represented by sticks). The ETGE motif is colored violet and pink, in the equilibrated and final structure, respectively. 